\title{
Multiple Integrals under Differential Constraints: Two-Scale Convergence and Homogenization
}

\author{
Irene Fonseca \\ Carnegie Mellon University, \\ fonseca@andrew.cmu.edu
}

\author{
Stefan Krömer \\ Carnegie Mellon University, \\ kroemers@andrew.cmu.edu
}

December 4, 2009

\begin{abstract}
Two-scale techniques are developed for sequences of maps $\left\{u_{k}\right\} \subset L^{p}\left(\Omega ; \mathbb{R}^{M}\right)$ satisfying a linear differential constraint $\mathcal{A} u_{k}=0$. These, together with $\Gamma$ convergence arguments and using the unfolding operator, provide a homogenization result for energies of the type

$$
F_{\varepsilon}(u):=\int_{\Omega} f\left(x, \frac{x}{\varepsilon}, u(x)\right) d x \text { with } u \in L^{p}\left(\Omega ; \mathbb{R}^{M}\right), \mathcal{A} u=0,
$$

that generalizes current results in the case where $\mathcal{A}=$ curl.

MSC 2000: 49J45, 35E99
\end{abstract}

\section{Introduction}

In this paper we study the limiting behavior of a family of energy functionals with periodic energy densities and underlying fields subject to differential constraints. We give an integral representation to

$$
\mathcal{F}(u):=\inf \left\{\begin{array}{l|l}
\liminf _{\varepsilon \rightarrow 0} \int_{\Omega} f\left(x, \frac{x}{\varepsilon}, u_{\varepsilon}\right) d x & \begin{array}{l}
u_{\varepsilon} \rightarrow u \text { in } L^{p}\left(\Omega ; \mathbb{R}^{M}\right), \\
\mathcal{A} u_{\varepsilon}=0
\end{array}
\end{array}\right\},
$$

where $N, M \in \mathbb{N}, \Omega \subset \mathbb{R}^{N}$ is an open, bounded set, $1<p<+\infty, u \in L^{p}\left(\Omega ; \mathbb{R}^{M}\right)$, $f: \Omega \times \mathbb{R}^{N} \times \mathbb{R}^{M} \rightarrow[0,+\infty)$ satisfies

(H0) $f(x, \cdot, \xi)$ is measurable for every $(x, \xi) \in \Omega \times \mathbb{R}^{M}$ and $f(\cdot, y, \cdot)$ is continuous for a.e. $y \in \mathbb{R}^{N}$;

(H1) $f(x, \cdot, \xi)$ is $Q$-periodic for every $(x, \xi) \in \Omega \times \mathbb{R}^{M}$, with $Q:=(0,1)^{N}$;

(H2) $\quad 0 \leq f(x, y, \xi) \leq C\left(1+|\xi|^{p}\right)$ for every $(x, \xi) \in \Omega \times \mathbb{R}^{M}$ and a.e. $y \in \mathbb{R}^{N}$,

and $\mathcal{A}$ is a first order partial differential operator of constant rank. Precisely, $\mathcal{A}$ maps $u=\left(u^{1}, \ldots, u^{M}\right): \Omega \rightarrow \mathbb{R}^{M}$ into $\mathcal{A} u=\left((\mathcal{A} u)^{1}, \ldots,(\mathcal{A} u)^{L}\right): \Omega \rightarrow \mathbb{R}^{L}, L \in \mathbb{N}$, with

$$
(\mathcal{A} u)^{l}:=\sum_{i=1}^{N} \sum_{m=1}^{M} A_{m}^{l i} \frac{\partial u^{m}}{\partial_{x_{i}}}, \quad l=1, \ldots, L,
$$


and the coefficients $A_{m}^{l i} \in \mathbb{R}$. The linear matrix valued function

$$
\mathbb{A}: \mathbb{R}^{N} \rightarrow \mathbb{R}^{L \times M}, \quad(\mathbb{A}(\xi))_{m}^{l}:=\sum_{i=1}^{N} A_{m}^{l i} \xi_{i}, \quad l=1, \ldots, L, m=1, \ldots, M,
$$

is related to $\mathcal{A}$ via the Fourier transform. We assume throughout that $\mathcal{A}$ satisfies Murat's condition of constant rank (see [23]), that is,

$$
\text { the rank of } \mathbb{A}(\xi) \in \mathbb{R}^{L \times M} \text { is constant as a function of } \xi \in \mathbb{R}^{N} \backslash\{0\} \text {. }
$$

The study of lowersemicontinuity and relaxation of energy functionals of this type was initiated by Dacorogna [13], followed by Fonseca and Müller [18], and also Braides, Fonseca and Leoni [7], among others. In the latter, the homogenization of a family of functionals as considered in [1] was studied, with $f$ independent of $x$, continuous in $y$ (note that in ( $\mathrm{H} 0)$ we only ask measurability), and coercive (note that no coercivity is required here in (H2)). Therefore, this work generalizes previous results in the variational approach of homogenization for $\mathcal{A}$-free fields. We recall that important examples that are included in this general setting are the case of divergence free fields, in which $\mathcal{A} u=0$ if and only if $\operatorname{div} u=0$, and the case of gradients, in which $\mathcal{A} u=0$ if and only if $\operatorname{curl} u=0$.

The main theorem of this paper is

Theorem 1.1. If (H0)-(H2) hold then for every $u \in L^{p}\left(\Omega ; \mathbb{R}^{M}\right)$ with $\mathcal{A} u=0$,

$$
\mathcal{F}(u)=\int_{\Omega} f_{\text {hom }}(x, u(x)) d x
$$

where, for $x \in \Omega$ and $\xi \in \mathbb{R}^{M}$,

$$
f_{\text {hom }}(x, \xi):=\liminf _{n \rightarrow \infty} \inf _{v \in \mathcal{V}_{\mathcal{A}}} \int_{Q} f(x, n y, \xi+v(y)) d y,
$$

and $\mathcal{V}_{\mathcal{A}}:=\left\{v \in L_{\mathrm{per}}^{p}\left(\mathbb{R}^{N} ; \mathbb{R}^{M}\right) \mid \int_{Q} v=0\right.$ and $\left.\mathcal{A} v=0\right\}$

The proof may be found in Section 3, and the tools used here are $\Gamma$-convergence, as it was introduced by De Giorgi (see [15] and [16]), the notion of two-scale convergence for $\mathcal{A}$-free sequences, introduced in the case of gradients by Nguetseng (see [22], [24] and [25]), further developed by Allaire and Briane (see [2] and [1]) and many other authors (see also [19]), and extended here to the general $\mathcal{A}$-free setting in Section 2. Further, to prove the lower bound

$$
\mathcal{F}(u) \geq \int_{\Omega} f_{\text {hom }}(x, u(x)) d x
$$

we use the unfolding operator as proposed by Cioranescu, Damlamian and Griso (see [11] and [12]; see also Visintin [26], [27]).

In Section 2 we develop the concept of two-scale convergence for $\mathcal{A}$-free fields, and in Theorem 2.12 we give a complete characterization of weak two-scale limits of $\mathcal{A}$-free sequences. Precisely, 
Theorem 1.2. A function $w \in L^{p}\left(\Omega ; L_{\mathrm{per}}^{p}\left(\mathbb{R}^{N} ; \mathbb{R}^{M}\right)\right)$ is the weak two-scale limit of a $\mathcal{A}$-free sequence $\left\{u_{\varepsilon}\right\} \subset L^{p}\left(\Omega ; \mathbb{R}^{M}\right)$ if and only if

$$
\mathcal{A}_{y_{0}} \bar{w}_{0}=0 \text { and } \mathcal{A}_{y_{1}} \bar{w}_{1}=0,
$$

where

$$
\bar{w}_{0}\left(y_{0}\right):=\int_{Q} w\left(y_{0}, y_{1}\right) d y_{1} \quad \text { and } \bar{w}_{1}\left(y_{0}, y_{1}\right):=w\left(y_{0}, y_{1}\right)-\bar{w}_{0}\left(y_{0}\right)
$$

for $y_{0} \in \Omega$ and $y_{1} \in Q$.

Recall that in the case of gradients, two-scale limits are of the form

$$
\left(y_{0}, y_{1}\right) \in \Omega \times Q \mapsto \nabla v_{0}\left(y_{0}\right)+\nabla_{y_{1}} v_{1}\left(y_{0}, y_{1}\right),
$$

with $v_{1} \in L^{p}\left(\Omega ; W_{\text {per }}^{1, p}(Q)\right)$ (see [1], and see [28] for a generalization). In this context, using this together with $\Gamma$-limit techniques, Baia and Fonseca in [4] obtained the integral representation for the limit energy of a family of functionals

$$
v \mapsto \int_{\Omega} f\left(x, \frac{x}{\varepsilon}, \nabla v_{\varepsilon}\right) d x
$$

just as in Theorem 1.1, under conditions (H0), (H1), and with (H2) strengthened with a $p$-coercivity condition. We remark that the result obtained in this paper now extends that in [4] to the case in which $f$ is not coercive. There is an extensive body of literature on homogenization of multiple integrals of the type (1.3), and in particular we refer to Braides and Defranceschi [6], Braides and Lukkassen [8], Lukkassen [21], Berlyand, Cioranescu and Golovaty [5], Babadjian and Baía [3], and the references therein.

\section{Weak two-scale limits for $\mathcal{A}$-free sequences}

Let $M, N \in \mathbb{N}$, let $1<p<\infty$, let $\Omega \subset \mathbb{R}^{N}$ be open and bounded and let $Q:=(0,1)^{N}$ be the unit cube in $\mathbb{R}^{N}$. In the following, spaces of functions in $\mathbb{R}^{N}$ which are $Q$ periodic are denoted using a subscript "per", where $u: \mathbb{R}^{N} \rightarrow \mathbb{R}^{M}$ is said to be $Q$-periodic if $u(x+\zeta)=f(x)$ for all $\zeta \in \mathbb{Z}^{N}$ and all $x \in \mathbb{R}^{N}$. In particular, we use the space

$$
L_{\text {per }}^{p}\left(\mathbb{R}^{N} ; \mathbb{R}^{M}\right):=\left\{u \in L_{\text {loc }}^{p}\left(\mathbb{R}^{N} ; \mathbb{R}^{M}\right) \mid u \text { is } Q \text {-periodic }\right\},
$$

endowed with the norm of $L^{p}\left(Q, \mathbb{R}^{M}\right)$.

Definition 2.1 (weak two-scale convergence [24], [2]). Given a function $w \in$ $L^{p}\left(\Omega ; L_{\text {per }}^{p}\left(\mathbb{R}^{N n} ; \mathbb{R}^{M}\right)\right)$ and a sequence $\left\{u_{\varepsilon}\right\}_{\varepsilon>0} \subset L^{p}\left(\Omega ; \mathbb{R}^{M}\right)$, we say that $\left\{u_{\varepsilon}\right\}$ weakly two-scale converges to $w$, or $u_{\varepsilon} \stackrel{2-s}{\longrightarrow} w$ in $L^{p}\left(\Omega ; \mathbb{R}^{M}\right)$ (with respect to the scales $x$ and $\left.\frac{x}{\varepsilon}\right)$, if

$$
\int_{\Omega} u_{\varepsilon}(x) \cdot \varphi\left(x, \frac{x}{\varepsilon}\right) d x \underset{\varepsilon \rightarrow 0}{\longrightarrow} \int_{\Omega} \int_{Q} w\left(y_{0}, y_{1}\right) \cdot \varphi\left(y_{0}, y_{1}\right) d y_{1} d y_{0},
$$

for every $\varphi \in L^{p^{\prime}}\left(\Omega ; C_{\text {per }}\left(\mathbb{R}^{N} ; \mathbb{R}^{M}\right)\right)$, where $p^{\prime}:=p /(p-1)$. 
Here and in the following, if we talk about a "sequence" with index $\varepsilon>0$, we understand that $\varepsilon$ can be replaced with an arbitrary sequence $\left(\varepsilon_{k}\right)_{k \in \mathbb{N}} \subset(0, \infty)$ such that $\varepsilon_{k} \rightarrow 0$ as $k \rightarrow \infty$. In particular, $u_{\varepsilon} \rightarrow u$ as $\varepsilon \rightarrow 0^{+}$(with respect to some notion of convergence) if and only if $u_{\varepsilon_{k}} \rightarrow u$ for every sequence $\left(\varepsilon_{k}\right)_{k \in \mathbb{N}} \subset(0, \infty)$ with $\varepsilon_{k} \rightarrow 0$ as $k \rightarrow \infty$.

Remark 2.2. Note that if $u_{\varepsilon} \stackrel{2-s}{\longrightarrow} w$ in $L^{p}\left(\Omega ; \mathbb{R}^{M}\right)$ then $u_{\varepsilon} \rightarrow \bar{w}_{0}$ in $L^{p}\left(\Omega ; \mathbb{R}^{M}\right)$, where $\bar{w}_{0}\left(y_{0}\right):=\int_{Q} w\left(y_{0}, y_{1}\right) d y_{1}$ for $y_{0} \in \Omega$.

Bounded sequences are compact with respect to two-scale weak convergence. Precisely (see [2], [22]):

Proposition 2.3. Every bounded sequence in $L^{p}\left(\Omega ; \mathbb{R}^{M}\right)$ has a subsequence which weakly two-scale converges to a limit in $L^{p}\left(\Omega ; L_{\mathrm{per}}^{p}\left(\mathbb{R}^{N} ; \mathbb{R}^{M}\right)\right)$.

A simple example of a weakly two-scale convergent sequence is addressed next:

Proposition 2.4. Given $u \in L^{p}\left(\Omega ; C_{\mathrm{per}}\left(\mathbb{R}^{N} ; \mathbb{R}^{M}\right)\right)$ or $u \in L_{\mathrm{per}}^{p}\left(\mathbb{R}^{N} ; C\left(\bar{\Omega} ; \mathbb{R}^{M}\right)\right)$ (the second variable being the periodic one), the sequence $\left\{u_{\varepsilon}\right\} \subset L^{p}\left(\Omega ; \mathbb{R}^{M}\right)$, with $u_{\varepsilon}(x):=$ $u\left(x, \frac{x}{\varepsilon}\right)$, is p-equiintegrable. It weakly two-scale converges to $u$, and it weakly converges in $L^{p}\left(\Omega ; \mathbb{R}^{M}\right)$ to $x \in \Omega \mapsto \int_{Q} u(x, y) d y$.

This result is an immediate consequence of the following lemma proved in [2] (see Lemma 5.2 and Corollary 5.4 in [2]).

Lemma 2.5. Let $g \in L^{1}\left(\Omega ; C_{\mathrm{per}}\left(\mathbb{R}^{N} ; \mathbb{R}^{M}\right)\right)$ or $g \in L_{\mathrm{per}}^{1}\left(\mathbb{R}^{N} ; C\left(\bar{\Omega} ; \mathbb{R}^{M}\right)\right)$. Then $\left\{g_{\varepsilon}\right\}$, with $g_{\varepsilon}(x):=g\left(x, \frac{x}{\varepsilon}\right)$ (the second variable being the periodic one), is a bounded, equiintegrable sequence in $L^{1}\left(\Omega ; \mathbb{R}^{M}\right)$ such that

$$
\int_{\Omega} g_{\varepsilon}(x) d x \rightarrow \int_{\Omega} \int_{Q} g(x, y) d x d y \quad \text { as } \varepsilon \rightarrow 0^{+} .
$$

Remark 2.6. Equiintegrability of $g_{\varepsilon}$ is not shown in [2], but it is a consequence of the following estimates: Let $E \subset \Omega$ be a measurable set. In the first case, i.e., if $g \in L^{1}\left(\Omega ; C_{\text {per }}\left(\mathbb{R}^{N} ; \mathbb{R}^{M}\right)\right)$, we have

$$
\int_{E}\left|g_{\varepsilon}(x)\right| d x \leq \int_{E} \sup _{y \in Q}|g(x, y)| d x d y .
$$

On the other hand, if $g \in L_{\text {per }}^{1}\left(\mathbb{R}^{N} ; C\left(\bar{\Omega} ; \mathbb{R}^{M}\right)\right)$, then

$$
\int_{E}\left|g_{\varepsilon}(x)\right| d x \leq \int_{E} \max _{x \in \bar{\Omega}}\left|g\left(x, \frac{y}{\varepsilon}\right)\right| d y .
$$

Note that since $\max _{x \in \bar{\Omega}}|g(x, \cdot)| \in L^{1}\left(Q ; \mathbb{R}^{M}\right),\left(\max _{x \in \bar{\Omega}}|g(x, \dot{\bar{\varepsilon}})|\right)_{\varepsilon}$ is a weakly convergent sequence in $L^{1}$ by the Riemann-Lebesgue lemma (see [17], e.g.), and thus equiintegrable.

Here, we study those two-scale weak limits which are generated by sequences $\left\{u_{\varepsilon}\right\}$ satisfying a differential constraint $\mathcal{A} u_{\varepsilon}=0$, where $\mathcal{A}$ denotes a homogeneous linear 
differential operator of first order mapping $u=\left(u^{1}, \ldots, u^{M}\right): \Omega \rightarrow \mathbb{R}^{M}$ into $\mathcal{A} u=$ $\left((\mathcal{A} u)^{1}, \ldots,(\mathcal{A} u)^{L}\right): \Omega \rightarrow \mathbb{R}^{L}$, with

$$
(\mathcal{A} u)^{l}:=\sum_{i=1}^{N} \sum_{m=1}^{M} A_{m}^{l i} \frac{\partial u^{m}}{\partial_{x_{i}}}, \quad l=1, \ldots, L,
$$

and the coefficients $A_{m}^{l i} \in \mathbb{R}$. Its formal adjoint is denoted by $\mathcal{A}^{*}$, which maps $v=$ $\left(v_{1}, \ldots, v_{L}\right): \Omega \rightarrow \mathbb{R}^{L}$ into $\mathcal{A}^{*} v: \Omega \rightarrow \mathbb{R}^{M}$, and is defined by

$$
\left(\mathcal{A}^{*} v\right)_{m}:=-\sum_{i=1}^{N} \sum_{l=1}^{L} A_{m}^{l i} \frac{\partial v_{l}}{\partial_{x_{i}}}, \quad m=1, \ldots, M
$$

If $u \in C_{c}^{1}\left(\Omega ; \mathbb{R}^{M}\right)$ and $v \in C_{c}^{1}\left(\Omega ; \mathbb{R}^{L}\right)$, or $u \in C_{\text {per }}^{1}\left(\mathbb{R}^{N} ; \mathbb{R}^{M}\right)$ and $v \in C_{\text {per }}^{1}\left(\mathbb{R}^{N} ; \mathbb{R}^{L}\right)$, then integration by parts yields

$$
\int_{\Omega} \mathcal{A} u \cdot v=\int_{\Omega} u \cdot \mathcal{A}^{*} v \text { or } \int_{Q} \mathcal{A} u \cdot v=\int_{Q} u \cdot \mathcal{A}^{*} v
$$

respectively.

Below, it is understood that if we apply $\mathcal{A}$ to a vector field depending on multiple variables, then the variable on which $\mathcal{A}$ operates is indicated as a subscript, e.g., $\mathcal{A}_{y} u(x, y)$ means that for the purpose of the application of $\mathcal{A}, u(x, y)$ is considered as a function of $y$ with $x$ being a fixed parameter. The linear matrix valued function

$$
\mathbb{A}: \mathbb{R}^{N} \rightarrow \mathbb{R}^{L \times M}, \quad(\mathbb{A}(\xi))_{m}^{l}:=\sum_{i=1}^{N} A_{m}^{l i} \xi_{i}, \quad l=1, \ldots, L, m=1, \ldots, M,
$$

is related to $\mathcal{A}$ via the Fourier transform. As a consequence of constant rank condition (1.2), the orthogonal projection $\mathbb{P}(\xi) \in \mathbb{R}^{M \times M}$ onto the kernel of $\mathbb{A}(\xi)$ in $\mathbb{R}^{M}$ is 0 homogeneous and continuous as a function of $\xi \in \mathbb{R}^{N} \backslash\{0\}$. We set $\mathbb{P}(0)$ to be the identity matrix in $\mathbb{R}^{N \times N}$. By the Hörmander-Mikhlin multiplier theorem, $\mathbb{P}$ gives rise to a continuous projection operator $\mathcal{P}: L_{\mathrm{per}}^{p}\left(\mathbb{R}^{N} ; \mathbb{R}^{M}\right) \rightarrow L_{\mathrm{per}}^{p}\left(\mathbb{R}^{N} ; \mathbb{R}^{M}\right)$ onto the kernel of $\mathcal{A}$,

$$
\mathcal{P}(u):=\mathcal{F}^{-1}(\mathbb{P} \mathcal{F}(u)),
$$

where $\mathcal{F}$ is the Fourier transform. It turns out that

$$
\|(I-\mathcal{P}) u\|_{L_{\mathrm{per}}^{p}\left(\mathbb{R}^{N} ; \mathbb{R}^{M}\right)} \leq C\|\mathcal{A} u\|_{W^{-1, p}\left(Q ; \mathbb{R}^{L}\right)} \text { for every } u \in L_{\mathrm{per}}^{p}\left(\mathbb{R}^{N} ; \mathbb{R}^{M}\right)
$$

for some constant $C>0$. For more details and a proof of (2.2), the reader is referred to [18].

Definition 2.7 (Notions of weak $\mathcal{A}$-differentiability and $\mathcal{A}$-free fields).

(i) If $u \in L^{p}\left(\Omega ; \mathbb{R}^{M}\right)$, then we say that $\mathcal{A} u$ exists in $L^{p}$ if there is a function $U \in L^{p}\left(\Omega ; \mathbb{R}^{L}\right)$ such that

$$
\int_{\Omega} u \cdot \mathcal{A}^{*} \varphi d y=\int_{\Omega} U \cdot \varphi d y \text { for every } \varphi \in C_{c}^{1}\left(\Omega ; \mathbb{R}^{L}\right) .
$$

In this case, we define $\mathcal{A} u:=U$. We say that $u$ is $\mathcal{A}$-free, or $\mathcal{A} u=0$, if the preceding equation is satisfied with $U=0$. 
(ii) If $v \in L_{\mathrm{per}}^{p}\left(\mathbb{R}^{N} ; \mathbb{R}^{M}\right)$, then we say that $\mathcal{A} v$ exists in $L_{\mathrm{per}}^{p}$, if there is a function $V \in L_{\mathrm{per}}^{p}\left(\mathbb{R}^{N} ; \mathbb{R}^{L}\right)$ such that

$$
\int_{Q} v \cdot \mathcal{A}^{*} \varphi d y=\int_{Q} V \cdot \varphi d y \text { for every } \varphi \in C_{\mathrm{per}}^{1}\left(\mathbb{R}^{N} ; \mathbb{R}^{L}\right) .
$$

In this case, we define $\mathcal{A} v:=V$. We say that $v$ is $\mathcal{A}$-free, or $\mathcal{A} v=0$, if the preceding equation is satisfied with $V=0$.

(iii) If $w \in L^{p}\left(\Omega ; L_{\text {per }}^{p}\left(\mathbb{R}^{N} ; \mathbb{R}^{M}\right)\right), w=w\left(y_{0}, y_{1}\right)$ with $\left(y_{0}, y_{1}\right) \in \Omega \times \mathbb{R}^{N}$ and $j \in$ $\{0,1\}$, then we say that $\mathcal{A}_{y_{j}} u$ exists in $L^{p}\left(\Omega ; L_{\mathrm{per}}^{p}\left(\mathbb{R}^{N} ; \mathbb{R}^{L}\right)\right)$ if there exists a function $W_{j} \in L^{p}\left(\Omega ; L_{\mathrm{per}}^{p}\left(\mathbb{R}^{N} ; \mathbb{R}^{L}\right)\right)$ such that

$$
\begin{array}{r}
\int_{\Omega} \int_{Q} w \cdot \mathcal{A}_{y_{j}}^{*} \varphi d y_{1} d y_{0}=\int_{\Omega} \int_{Q} W_{j} \cdot \varphi d y_{1} d y_{0} \\
\text { for every } \varphi \in C_{c}^{1}\left(\Omega ; C_{\mathrm{per}}^{1}\left(\mathbb{R}^{N} ; \mathbb{R}^{L}\right)\right),
\end{array}
$$

In this case, we define $\mathcal{A}_{y_{j}} w:=W_{j}$.

The following extension result plays an important role in the variational theory of $\mathcal{A}$-free fields (see also [18]):

Lemma 2.8 ( $\mathcal{A}$-free periodic extension). Let $D \subset Q:=(0,1)^{N} \subset \mathbb{R}^{N}$ be open, let $1<p<\infty$ and let $\mathcal{A}$ satisfy (1.2). Then for every p-equiintegrable sequence $\left\{v_{n}\right\} \subset L^{p}\left(D ; \mathbb{R}^{M}\right)$ with $v_{n} \rightarrow 0$ in $L^{p}\left(D ; \mathbb{R}^{M}\right)$ and $\mathcal{A} v_{n} \rightarrow 0$ in $W^{-1, p}\left(D ; \mathbb{R}^{L}\right)$, there exists an $\mathcal{A}$-free sequence $\left\{u_{n}\right\} \subset L_{\mathrm{per}}^{p}\left(\mathbb{R}^{N} ; \mathbb{R}^{M}\right)$, p-equiintegrable in $Q$, such that

$$
u_{n}-v_{n} \rightarrow 0 \text { in } L^{p}\left(D ; \mathbb{R}^{M}\right), \quad u_{n} \rightarrow 0 \text { in } L^{p}\left(Q \backslash D ; \mathbb{R}^{M}\right), \quad \int_{Q} u_{n}(x)=0,
$$

and $\left\|u_{n}\right\|_{L^{p}\left(Q ; \mathbb{R}^{M}\right)} \leq C\left\|v_{n}\right\|_{L^{p}\left(d ; \mathbb{R}^{M}\right)}$ for all $n \in \mathbb{N}$ and some $C=C(\mathcal{A})>0$.

Proof. For every $k \in \mathbb{N}$ choose $\varphi_{k} \in C_{c}^{\infty}(D ;[0,1])$ such that $\varphi_{k}(x)=1$ whenever $\operatorname{dist}\left(x ; \mathbb{R}^{N} \backslash D\right) \geq \frac{1}{k}$. Clearly,

$$
\mathcal{A}\left(\varphi_{k} v_{n}\right)=\varphi_{k} \mathcal{A} v_{n}+\sum_{i=1}^{N} \sum_{m=1}^{M} v_{n}^{m} A_{m}^{l i} \partial_{i} \varphi_{k} \underset{n \rightarrow \infty}{\longrightarrow} 0 \text { in } W^{-1, p}\left(Q ; \mathbb{R}^{M}\right),
$$

for fixed $k$, since $v_{n} \rightarrow 0$ in $L^{p}$ and $L^{p}$ is compactly embedded in $W^{-1, p}$. Hence, we may choose a sequence $k(n) \rightarrow \infty$ such that

$$
\mathcal{A}\left(\varphi_{k(n)} v_{n}\right) \underset{n \rightarrow \infty}{\longrightarrow} 0 \text { in } W^{-1, p}\left(Q ; \mathbb{R}^{M}\right) .
$$

For each $n, \varphi_{k(n)} v_{n}$ can be considered as an element of $L_{\mathrm{per}}^{p}\left(\mathbb{R}^{N} ; \mathbb{R}^{M}\right)$ by extending it to $\mathbb{R}^{N} Q$-periodically. Let

$$
\tilde{u}_{n}:=\mathcal{P}\left(\varphi_{k(n)} v_{n}\right) .
$$

Then $\tilde{u}_{n} \in L_{\text {per }}^{p}\left(\mathbb{R}^{N} ; \mathbb{R}^{M}\right)$, and by $(2.2)$,

$$
\left\|\tilde{u}_{n}\right\|_{L^{p}\left(Q ; \mathbb{R}^{M}\right)}=\left\|\mathcal{P}\left(\varphi_{k(n)} v_{n}\right)\right\|_{L^{p}\left(Q ; \mathbb{R}^{M}\right)} \leq C\left\|\varphi_{k(n)} v_{n}\right\|_{L^{p}\left(Q ; \mathbb{R}^{M}\right)} \leq C\left\|v_{n}\right\|_{L^{p}\left(D ; \mathbb{R}^{M}\right)}
$$


by the continuity of $\mathcal{P}$ on $L_{\mathrm{per}}^{p}$. Also, since $\left\{\left|v_{n}\right|^{p}\right\}$ is equiintegrable, we have

$$
\begin{aligned}
\left\|\tilde{u}_{n}-v_{n}\right\|_{L^{p}\left(D ; \mathbb{R}^{M}\right)} & \leq\left\|\mathcal{P}\left(\varphi_{k(n)} v_{n}\right)-\varphi_{k(n)} v_{n}\right\|_{L^{p}\left(Q ; \mathbb{R}^{M}\right)}+\left\|\left(1-\varphi_{k(n)}\right) v_{n}\right\|_{L^{p}\left(D ; \mathbb{R}^{M}\right)} \\
& \leq C\left\|\mathcal{A}\left(\varphi_{k(n)} v_{n}\right)\right\|_{W^{-1, p}\left(Q ; \mathbb{R}^{L}\right)}+\left\|\left(1-\varphi_{k(n)}\right) v_{n}\right\|_{L^{p}\left(D ; \mathbb{R}^{M}\right)} \underset{n \rightarrow \infty}{\longrightarrow} 0
\end{aligned}
$$

and

$$
\begin{aligned}
\left\|\tilde{u}_{n}\right\|_{L^{p}\left(Q \backslash D ; \mathbb{R}^{M}\right)} & =\left\|\mathcal{P}\left(\varphi_{k(n)} v_{n}\right)-\varphi_{k(n)} v_{n}\right\|_{L^{p}\left(Q \backslash D ; \mathbb{R}^{M}\right)} \\
& \leq C\left\|\mathcal{A}\left(\varphi_{k(n)} v_{n}\right)\right\|_{W^{-1, p}\left(Q ; \mathbb{R}^{L}\right)} \underset{n \rightarrow \infty}{\longrightarrow} 0 .
\end{aligned}
$$

To verify the $p$-equiintegrability of $\left\{\tilde{u}_{n}\right\}$ in $Q$ let $E \subset Q$, and observe that

$$
\begin{aligned}
\int_{E}\left|\tilde{u}_{n}\right|^{p} & \leq C \int_{E}\left|\mathcal{P}\left(\varphi_{k(n)} v_{n}\right)-\varphi_{k(n)} v_{n}\right|^{p}+C \int_{E \cap D}\left|v_{n}\right|^{p} \\
& \leq C\left\|\mathcal{A}\left(\varphi_{k(n)} v_{n}\right)\right\|_{W^{-1, p}\left(Q ; \mathbb{R}^{L}\right)}^{p}+C \int_{E \cap D}\left|v_{n}\right|^{p} .
\end{aligned}
$$

It suffices to set $u_{n}(x):=\tilde{u}_{n}(x)-\int_{Q} \tilde{u}_{n}(y) d y$.

The following condition turns to be the characterization of weak two-scale limits of $\mathcal{A}$-free sequences (see Theorem 2.12 below).

Definition 2.9 (generalized $\mathcal{A}$-free fields with one microscale). We say that $w \in$ $L^{p}\left(\Omega ; L_{\mathrm{per}}^{p}\left(\mathbb{R}^{N} ; \mathbb{R}^{M}\right)\right)$ is generalized $\mathcal{A}$-free if

$$
\mathcal{A}_{y_{0}} \bar{w}_{0}=0 \text { and } \mathcal{A}_{y_{1}} \bar{w}_{1}=0
$$

where $\bar{w}_{0} \in L^{p}\left(\Omega ; \mathbb{R}^{M}\right)$ and $\bar{w}_{1} \in L^{p}\left(\Omega ; L_{\text {per }}^{p}\left(\mathbb{R}^{N} ; \mathbb{R}^{M}\right)\right)$ are defined by

$$
\bar{w}_{0}\left(y_{0}\right):=\int_{Q} w\left(y_{0}, y_{1}\right) d y_{1} \text { and } \bar{w}_{1}\left(y_{0}, y_{1}\right):=w\left(y_{0}, y_{1}\right)-\bar{w}_{0}\left(y_{0}\right)
$$

for $y_{0} \in \Omega$ and $y_{1} \in Q$.

Proposition 2.10. Let $\left\{u_{\varepsilon}\right\}$ be a bounded, $\mathcal{A}$-free sequence in $L^{p}\left(\Omega ; \mathbb{R}^{M}\right)$ which weakly two-scale converges to a function $w \in L^{p}\left(\Omega ; L_{\mathrm{per}}^{p}\left(\mathbb{R}^{N} ; \mathbb{R}^{M}\right)\right)$. Then $w$ is generalized $\mathcal{A}$-free.

Proof. Fix $\psi \in C_{c}^{1}\left(\Omega ; \mathbb{R}^{M}\right)$. We have

$$
\begin{aligned}
0=\lim _{\varepsilon \rightarrow 0^{+}} \int_{\Omega} u_{\varepsilon}(x) \cdot \mathcal{A}^{*} \psi(x) d x & =\int_{\Omega} \int_{Q} w\left(y_{0}, y_{1}\right) \cdot \mathcal{A}^{*} \psi\left(y_{0}\right) d y_{1} d y_{0} \\
& =\int_{\Omega} \bar{w}_{0} \cdot \mathcal{A}^{*} \psi\left(y_{0}\right) d y_{0}
\end{aligned}
$$

where we used the facts that $u_{\varepsilon}$ is $\mathcal{A}$-free, (2.1) and $(2.4)_{1}$, in this order. This establishes $(2.3)_{1}$. Next, define $\varphi\left(y_{0}, y_{1}\right):=\psi\left(y_{0}\right) \phi\left(y_{1}\right)$ for arbitrary functions 
$\psi \in C_{c}^{1}\left(\Omega ; \mathbb{R}^{N}\right)$ and $\phi \in C_{\text {per }}^{1}\left(\mathbb{R}^{N} ; \mathbb{R}^{L}\right)$. Since $u_{\varepsilon}$ is $\mathcal{A}$-free, we have that

$$
\begin{aligned}
0 & =\lim _{\varepsilon \rightarrow 0^{+}} \int_{\Omega} u_{\varepsilon}(x) \cdot \varepsilon \mathcal{A}^{*}\left[\varphi\left(x, \frac{x}{\varepsilon}\right)\right] d x \\
& =\lim _{\varepsilon \rightarrow 0^{+}} \int_{\Omega} u_{\varepsilon}(x) \cdot\left[\varepsilon\left(\mathcal{A}^{*} \psi\right)(x) \phi\left(\frac{x}{\varepsilon}\right)+\psi(x)\left(\mathcal{A}^{*} \phi\right)\left(\frac{x}{\varepsilon}\right)\right] d x \\
& =\int_{\Omega} \int_{Q} w\left(y_{0}, y_{1}\right) \cdot \psi\left(y_{0}\right) \mathcal{A}_{y_{1}}^{*} \phi\left(y_{1}\right) d y_{1} d y_{0} \\
& =\int_{\Omega}\left(\int_{Q} \bar{w}_{1} \cdot \mathcal{A}_{y_{1}}^{*} \phi\left(y_{1}\right) d y_{1}\right) \psi\left(y_{0}\right) d y_{0},
\end{aligned}
$$

where we used the fact that $\int_{Q} \mathcal{A}_{y_{1}}^{*} \phi\left(y_{1}\right) d y_{1}=0$. This yields $(2.3)_{2}$.

In the case of gradients (i.e., curl-free fields) and divergence-free fields, (2.3) is known to characterize the weak two-scale limits of $\mathcal{A}$-free sequences in $L^{p}$ (see [1] and [2]). Precisely, weak two-scale limits of gradients of $W^{1, p}(\Omega)$-bounded functions are all functions of the form $\left(y_{0}, y_{1}\right) \mapsto \nabla_{y_{0}} u_{0}\left(y_{0}\right)+\nabla_{y_{1}} u_{1}\left(y_{0}, y_{1}\right)$ with $u_{0} \in W^{1, p}(\Omega), u_{1} \in$ $L^{p}\left(\Omega ; W_{\text {per }}^{1, p}\left(\mathbb{R}^{N}\right)\right)$, and weak two-scale limits of divergence free fields in $L^{p}\left(\Omega ; \mathbb{R}^{N}\right)$ are all functions $w \in L^{p}\left(\Omega ; L_{\text {per }}^{p}\left(\mathbb{R}^{N} ; \mathbb{R}^{N}\right)\right)$ such that $\operatorname{div}_{y_{0}} \bar{w}_{0}=0$ and $\operatorname{div}_{y_{1}} \bar{w}_{1}\left(y_{0}, y_{1}\right)=0$. To prove the corresponding result for a general $\mathcal{A}$ satisfying (1.2), we reconstruct a suitable $\mathcal{A}$-free sequence from a given generalized $\mathcal{A}$-free function.

Proposition 2.11. Let $Y \subset \mathbb{R}^{N}$ be an open cube compactly containing $\Omega$, and suppose that $\mathcal{A}$ satisfies (1.2). Then for every $w \in L^{p}\left(\Omega ; L_{\mathrm{per}}^{p}\left(\mathbb{R}^{N} ; \mathbb{R}^{M}\right)\right), w=w\left(y_{0}, y_{1}\right)$, such that $\int_{Q} w\left(\cdot, y_{1}\right) d y_{1}=0$ (i.e., $w=\bar{w}_{1}$ and $\bar{w}_{0}=0$ ) and $\mathcal{A}_{y_{1}} w=0$, there exists an $\mathcal{A}$-free, p-equiintegrable sequence $\left\{u_{\varepsilon}\right\} \subset L^{p}\left(Y ; \mathbb{R}^{M}\right)$ such that the restriction of $u_{\varepsilon}$ to $\Omega$ weakly two-scale converges to $w$ and $u_{\varepsilon} \rightarrow 0$ weakly in $L^{p}\left(Y ; \mathbb{R}^{M}\right)$.

Proof. Step 1: Suppose first that $w \in C^{1}\left(\mathbb{R}^{N} ; C_{\mathrm{per}}^{1}\left(\mathbb{R}^{N} ; \mathbb{R}^{M}\right)\right)$. Define

$$
v_{\varepsilon}(x):=\int_{Q} w\left(x+\varepsilon y, \frac{x}{\varepsilon}\right) d y, x \in \bar{\Omega} .
$$

Then $v_{\varepsilon} \in C^{1}\left(\bar{\Omega} ; \mathbb{R}^{M}\right)$. By Hölder's inequality, we have

$$
\left|v_{\varepsilon}(x)\right|^{p} \leq \int_{Q}\left|w\left(x+\varepsilon y, \frac{x}{\varepsilon}\right)\right|^{p} d y,
$$

and by the volume-preserving change of variables

$$
S_{\varepsilon}: \mathbb{R}^{N} \times Q \rightarrow \mathbb{R}^{N} \times \bar{Q}, \quad S_{\varepsilon}(x, y):=\left(x+\varepsilon y, \frac{x}{\varepsilon}-\left\lfloor\frac{x}{\varepsilon}\right\rfloor\right),
$$

we get the bound

$$
\left\|v_{\varepsilon}\right\|_{L^{p}\left(E ; \mathbb{R}^{M}\right)} \leq\|w\|_{L^{p}\left(S_{\varepsilon}(E \times Q) ; \mathbb{R}^{M}\right)} \leq\|w\|_{L^{p}\left(E_{\varepsilon} \times Q ; \mathbb{R}^{M}\right)}
$$

for every measurable $E \subset \Omega$, with $E_{\varepsilon}:=\left\{x \in \mathbb{R}^{N} \mid \operatorname{dist}(x ; E)<\varepsilon\right\}$. In particular, $\left\{v_{\varepsilon}\right\}$ is $p$-equiintegrable in $\Omega$. Next, we claim that

$$
\begin{aligned}
& v_{\varepsilon} \stackrel{2-s}{\rightarrow} w \text { in } L^{p}\left(\Omega ; \mathbb{R}^{M}\right), \\
& v_{\varepsilon} \rightarrow 0 \text { in } L^{p}\left(\Omega ; \mathbb{R}^{M}\right) \text { and } \mathcal{A} v_{\varepsilon} \rightarrow 0 \text { strongly in } W^{-1, p}\left(\Omega ; \mathbb{R}^{L}\right) .
\end{aligned}
$$


To prove $(2.6)$, we set $\tilde{v}_{\varepsilon}(x):=w\left(x, \frac{x}{\varepsilon}\right)$. Clearly, $\left\{\tilde{v}_{\varepsilon}\right\} \subset C^{1}\left(\mathbb{R}^{N} ; \mathbb{R}^{M}\right)$ is locally bounded in $L^{\infty}$. Since $\mathcal{A}_{y_{1}} w=0$, we have

$$
\mathcal{A} \tilde{v}_{\varepsilon}(x)-\mathcal{A} v_{\varepsilon}(x)=\int_{Q}\left(\mathcal{A}_{y_{0}} w\left(x, \frac{x}{\varepsilon}\right)-\mathcal{A}_{y_{0}} w\left(x+\varepsilon y, \frac{x}{\varepsilon}\right)\right) d y
$$

and thus $\mathcal{A} v_{\varepsilon}-\mathcal{A} \tilde{v}_{\varepsilon} \rightarrow 0$ in $L^{p}\left(\Omega ; \mathbb{R}^{M}\right)$, where we used the fact that $\mathcal{A}_{y_{0}} w$ is uniformly continuous in $\Omega_{1} \times \mathbb{R}^{N}$, with $\Omega_{1}:=\left\{x \in \mathbb{R}^{N} \mid \operatorname{dist}(x ; \Omega)<1\right\}$. In addition, by Proposition 2.4 we obtain

$$
\mathcal{A} \tilde{v}_{\varepsilon}=\mathcal{A}_{y_{0}} w(\cdot, \dot{\bar{\varepsilon}}) \underset{\varepsilon \rightarrow 0^{+}}{\longrightarrow} \int_{Q} \mathcal{A}_{y_{0}} w\left(\cdot, y_{1}\right) d y_{1}=\mathcal{A} \int_{Q} w\left(\cdot, y_{1}\right) d y_{1}=0
$$

weakly in $L^{p}\left(\Omega ; \mathbb{R}^{M}\right)$. By (2.7) and (2.8), we deduce that $\lim _{\varepsilon} \mathcal{A} v_{\varepsilon}=0$ weakly in $L^{p}\left(\Omega ; \mathbb{R}^{L}\right)$, and thus strongly in $W^{-1, p}\left(\Omega ; \mathbb{R}^{L}\right)$ by Sobolev's compact embedding theorem. Again using the uniformly continuity of $w$ in $\Omega_{1} \times \mathbb{R}^{N}$, we have that $v_{\varepsilon}-\tilde{v}_{\varepsilon} \rightarrow 0$ in $L^{p}\left(\Omega ; \mathbb{R}^{M}\right)$ and, in particular, $v_{\varepsilon} \stackrel{2-s}{\rightarrow} w$ and $v_{\varepsilon} \rightarrow 0$ in $L^{p}\left(\Omega ; \mathbb{R}^{M}\right)$ (note that $\bar{w}_{0}=0$ ). This completes the proof of (2.6), which now allows us to apply Lemma 2.8 to $\left\{v_{\varepsilon}\right\}$ (with $D:=\Omega$ and $Y$ in place of $Q$, if necessary translate and rescale). We thus get an $\mathcal{A}$-free, $p$-equiintegrable sequence $\left\{u_{\varepsilon}\right\} \subset L^{p}\left(Y ; \mathbb{R}^{M}\right)$ such that $v_{\varepsilon}-u_{\varepsilon} \rightarrow 0$ in $L^{p}\left(\Omega ; \mathbb{R}^{M}\right)$ and $u_{\varepsilon} \rightarrow 0$ in $L^{p}\left(Y \backslash \Omega ; \mathbb{R}^{M}\right)$. In particular, $\left\{u_{\varepsilon}\right\}$ weakly two-scale converges to $w$ in $\Omega$ and weakly converges to zero in $Y$. In addition, in view of (2.5) we obtain

$$
\begin{aligned}
\left\|u_{\varepsilon}\right\|_{L^{p}\left(E ; \mathbb{R}^{M}\right)} & \leq\left\|u_{\varepsilon}\right\|_{L^{p}\left(Y \backslash \Omega ; \mathbb{R}^{M}\right)}+\left\|u_{\varepsilon}-v_{\varepsilon}\right\|_{L^{p}\left(E \cap \Omega ; \mathbb{R}^{M}\right)}+\left\|v_{\varepsilon}\right\|_{L^{p}\left(E \cap \Omega ; \mathbb{R}^{M}\right)} \\
& \leq \sigma_{\varepsilon}+\|w\|_{L^{p}\left(S_{\varepsilon}((E \cap \Omega) \times Q) ; \mathbb{R}^{M}\right)},
\end{aligned}
$$

for every $0<\varepsilon \leq 1$ and every measurable $E \subset Y$, where

$$
\sigma_{\varepsilon}:=\left\|u_{\varepsilon}\right\|_{L^{p}\left(Y \backslash \Omega ; \mathbb{R}^{M}\right)}+\left\|u_{\varepsilon}-v_{\varepsilon}\right\|_{L^{p}\left(\Omega ; \mathbb{R}^{M}\right)} \rightarrow 0 \text { as } \varepsilon \rightarrow 0^{+} .
$$

Consequently, $\left\{u_{\varepsilon}\right\}$ is $p$-equiintegrable in $Y$.

Step 2: We use a mollification and a diagonalization argument to reduce the general case to the previous step. For every $y_{1} \in \mathbb{R}^{N}$ extend $w\left(\cdot, y_{1}\right)$ by zero outside $\Omega$, whence $\mathcal{A}_{y_{1}} w=0$ in $\mathbb{R}^{N} \times \mathbb{R}^{N}$ and $\int_{Q} w\left(\cdot, y_{1}\right) d y_{1}=0$ in $\mathbb{R}^{N}$. Standard mollification of $w=w\left(y_{0}, y_{1}\right)$ by convolution (twice, first in $y_{1}$ and then in $y_{0}$ ) yields a sequence $\left\{w_{j}\right\} \subset C_{c}^{\infty}\left(\mathbb{R}^{N} ; C_{\text {per }}^{\infty}\left(\mathbb{R}^{N} ; \mathbb{R}^{M}\right)\right)$ such that $\left\|w_{j}\right\|_{L^{p}\left(\mathbb{R}^{N} ; L^{p}\left(Q ; \mathbb{R}^{M}\right)\right)} \leq\|w\|_{L^{p}\left(\Omega ; L^{p}\left(Q ; \mathbb{R}^{M}\right)\right)}$, $w_{j} \rightarrow w$ in $L^{p}$, and each $w_{j}$ satisfies satisfies

$$
\mathcal{A}_{y_{1}} w_{j}=0 \text { in } \mathbb{R}^{N} \times \mathbb{R}^{N} \text { and } \int_{Q} w_{j}\left(\cdot, y_{1}\right) d y_{1}=0 \text { in } \mathbb{R}^{N},
$$

since both properties are invariant under convolution in $y_{1}$. As to the latter, note that for arbitrary $z \in \mathbb{R}^{N}, \int_{Q} w\left(\cdot, z+y_{1}\right) d y_{1}=\int_{Q} w\left(\cdot, y_{1}\right) d y_{1}=0$, since $w$ is $Q$-periodic in $y_{1}$.

By Step 1, for each $j$ there exists an $\mathcal{A}$-free sequence $\left\{u_{j, \varepsilon}\right\} \subset L^{p}\left(Y ; \mathbb{R}^{M}\right)$ such that

$$
u_{j, \varepsilon} \stackrel{2-s}{\longrightarrow} w_{j} \text { in } L^{p}\left(\Omega ; \mathbb{R}^{M}\right) \text { and } u_{j, \varepsilon} \rightarrow 0 \text { in } L^{p}\left(Y ; \mathbb{R}^{M}\right)
$$


as $\varepsilon \rightarrow 0^{+}$. Moreover, by (2.9),

$$
\left\|u_{j, \varepsilon}\right\|_{L^{p}\left(E ; \mathbb{R}^{M}\right)} \leq \sigma_{j, \varepsilon}+\left\|w_{j}\right\|_{L^{p}\left(S_{\varepsilon}((E \cap \Omega) \times Q) ; \mathbb{R}^{M}\right)}
$$

for every measurable $E \subset Y$, with an error term satisfying $\sigma_{j, \varepsilon} \rightarrow 0$ as $\varepsilon \rightarrow 0^{+}$for fixed $j$. Further,

$$
\left\|w_{j}\right\|_{L^{p}\left(S_{\varepsilon}((E \cap \Omega) \times Q) ; \mathbb{R}^{M}\right)} \leq\left\|w_{j}-w\right\|_{L^{p}\left(\mathbb{R}^{N} \times Q ; \mathbb{R}^{M}\right)}+\|w\|_{L^{p}\left(S_{\varepsilon}((E \cap \Omega) \times Q) ; \mathbb{R}^{M}\right)} .
$$

Since the spaces of test functions for weak two-scale convergence and weak convergence in $L^{p}$, i.e., $L^{p^{\prime}}\left(\Omega ; C_{\text {per }}\left(\mathbb{R}^{N} ; \mathbb{R}^{M}\right)\right)$ and $L^{p^{\prime}}\left(\Omega ; \mathbb{R}^{M}\right)$, repectively, are both separable, a diagonalizing argument yields an integer valued function $\varepsilon \mapsto j(\varepsilon)$ such that $j(\varepsilon) \rightarrow \infty$, the sequence $\left\{u_{\varepsilon}\right\}$, with $u_{\varepsilon}:=u_{j(\varepsilon), \varepsilon}$, weakly two-scale converges to $w$ in $\Omega$ and weakly converges to zero in $Y$, and $\sigma_{j(\varepsilon), \varepsilon} \rightarrow 0$ as $\varepsilon \rightarrow 0^{+}$. In particular, (2.10) and (2.11) imply that $u_{\varepsilon}$ is $p$-equiintegrable on $Y$.

We conclude that $\mathcal{A}$-free weak two scale limits are characterized as follows.

Theorem 2.12. Let $1<p<\infty$, let $\Omega \subset \mathbb{R}^{N}$ be open and bounded, and suppose that $\mathcal{A}$ satisfies (1.2). Then $w \in L^{p}\left(\Omega ; L_{\mathrm{per}}^{p}\left(\mathbb{R}^{N} ; \mathbb{R}^{M}\right)\right)$ is the weak two-scale limit of some bounded, $\mathcal{A}$-free sequence $\left\{u_{\varepsilon}\right\} \subset L^{p}\left(\Omega ; \mathbb{R}^{M}\right)$ if and only if $w$ is generalized $\mathcal{A}$-free.

Proof. By Proposition 2.10, if $w$ is the weak two-scale limit of an $\mathcal{A}$-free sequence then $w$ is generalized $\mathcal{A}$-free. Conversely, given a generalized $\mathcal{A}$-free $w$, we apply Proposition 2.11 to $\bar{w}_{1}$ (using the notation of Definition 2.9). This yields an $\mathcal{A}$-free, bounded sequence $\left\{\tilde{u}_{\varepsilon}\right\}$ weakly two-scale converging to $\bar{w}_{1}$. Hence, $u_{\varepsilon}:=\bar{w}_{0}+\tilde{u}_{\varepsilon}$ weakly two-scale converges to $w=\bar{w}_{0}+\bar{w}_{1}$.

\section{$3 \mathcal{A}$-free homogenization with one microscale}

Let $\Omega \subset \mathbb{R}^{N}$ be open and bounded, let $1<p<\infty$ and $\varepsilon \in(0,1]$, and consider the functional

$$
F_{\varepsilon}(u):=\int_{\Omega} f\left(x, \frac{x}{\varepsilon}, u(x)\right) d x \text { for } u \in \mathcal{U}_{\mathcal{A}}:=\left\{u \in L^{p}\left(\Omega ; \mathbb{R}^{M}\right) \mid \mathcal{A} u=0\right\},
$$

where $f: \Omega \times \mathbb{R}^{N} \times \mathbb{R}^{M} \rightarrow \mathbb{R}$ satisfies the conditions (H0)-(H2) listed in the introduction.

Our main result is the following:

Theorem 3.1. If $\mathcal{A}$ satisfies (1.2) and (HO)-(H2) hold, then

$$
F_{\text {hom }}(u):=\Gamma-\lim _{\varepsilon \rightarrow 0^{+}} F_{\varepsilon}(u),
$$

the $\Gamma$-limit in the sense of De Giorgi with respect to weak convergence in $L^{p}$, exists for every $u \in \mathcal{U}_{\mathcal{A}}$. Moreover,

$$
F_{\text {hom }}(u)=\liminf _{n \rightarrow \infty} \inf _{w \in \mathcal{W}_{\mathcal{A}}} \int_{\Omega} \int_{Q} f(x, n y, u(x)+w(x, y)) d y d x,
$$

where $\mathcal{W}_{\mathcal{A}}:=\left\{w \in L^{p}\left(\Omega ; L_{\mathrm{per}}^{p}\left(\mathbb{R}^{N} ; \mathbb{R}^{M}\right)\right) \mid \int_{Q} w(\cdot, y) d y=0, \mathcal{A}_{y} w=0\right\}$ 
We recall that the $\Gamma\left(L^{p}\right.$-weak $)$-limit of $F_{\varepsilon}$ exists at $u$ if

$$
\begin{aligned}
& \Gamma-\liminf F_{\varepsilon}(u):=\inf \left\{\liminf F_{\varepsilon}\left(u_{\varepsilon}\right) \mid\left\{u_{\varepsilon}\right\} \subset \mathcal{U}_{\mathcal{A}}, u_{\varepsilon} \rightarrow u \text { in } L^{p}\left(\Omega ; \mathbb{R}^{M}\right)\right\}, \\
& \Gamma-\lim \sup F_{\varepsilon}(u):=\inf \left\{\limsup F_{\varepsilon}\left(u_{\varepsilon}\right) \mid\left\{u_{\varepsilon}\right\} \subset \mathcal{U}_{\mathcal{A}}, u_{\varepsilon} \rightarrow u \text { in } L^{p}\left(\Omega ; \mathbb{R}^{M}\right)\right\}
\end{aligned}
$$

coincide (see [14]). The corollary below provides an integral representation for $F_{\text {hom }}$.

Corollary 3.2. Under the asssumptions of Theorem 3.1, if $u \in \mathcal{U}_{\mathcal{A}}$ then

$$
F_{\text {hom }}(u)=\int_{\Omega} f_{\text {hom }}(x, u(x)) d x
$$

where for $x \in \Omega$ and $\xi \in \mathbb{R}^{M}$,

$$
f_{\text {hom }}(x, \xi):=\liminf _{n \rightarrow \infty} \inf _{v \in \mathcal{V}_{\mathcal{A}}} \int_{Q} f(x, n y, \xi+v(y)) d y
$$

and $\mathcal{V}_{\mathcal{A}}:=\left\{v \in L_{\mathrm{per}}^{p}\left(\mathbb{R}^{N} ; \mathbb{R}^{M}\right) \mid \int_{Q} v=0\right.$ and $\left.\mathcal{A} v=0\right\}$

Remark 3.3. For $n \in \mathbb{N}, u \in \mathcal{U}_{\mathcal{A}}, w \in \mathcal{W}_{\mathcal{A}}, v \in \mathcal{V}_{\mathcal{A}}$ and $\xi \in \mathbb{R}^{N}$ define

$$
\begin{aligned}
& \hat{F}(n, u, w):=\int_{\Omega} \int_{Q} f(x, n y, u(x)+w(x, y)) d y d x, \\
& \hat{f}(n, x, \xi, v):=\int_{Q} f(x, n y, \xi+v(y)) d y
\end{aligned}
$$

Then for every $k, n \in \mathbb{N}$ we have that

$$
\begin{aligned}
& \inf _{w \in \mathcal{W}_{\mathcal{A}}} \hat{F}(k n, u, w) \leq \inf _{w \in \mathcal{W}_{\mathcal{A}}} \hat{F}(n, u, w) \text { for every } u \in \mathcal{U}_{\mathcal{A}}, \text { and } \\
& \inf _{v \in \mathcal{V}_{\mathcal{A}}} \hat{f}(k n, x, \xi, v) \leq \inf _{v \in \mathcal{V}_{\mathcal{A}}} \hat{f}(n, x, \xi, v) \text { for every }(x, \xi) \in \Omega \times \mathbb{R}^{M} .
\end{aligned}
$$

Indeed, if $w \in \mathcal{W}_{\mathcal{A}}$ then $\bar{w}(x, y):=w(x, k y) \in \mathcal{W}_{\mathcal{A}}$, and (H1) together with the periodicity of $w(x, \cdot)$ and a change of variables yield $\hat{F}(k n, u, \bar{w})=\hat{F}(n, u, w)$. Similarly, if $v \in \mathcal{V}_{\mathcal{A}}$ then $\bar{v}(y):=v(k y) \in \mathcal{V}_{\mathcal{A}}$ and $\hat{f}(k n, x, \xi, \bar{v})=\hat{f}(n, x, \xi, v)$. In particular, $n \mapsto \inf _{w \in \mathcal{W}_{\mathcal{A}}} \hat{F}(n !, u, w)$ is decreasing, $\hat{F}(n !, u, w) \leq \hat{F}(n, u, w)$ and so $\lim _{n} \inf _{w \in \mathcal{W}_{\mathcal{A}}} \hat{F}(n !, u, w)$ exists. Therefore,

$$
\begin{aligned}
& \lim _{n \rightarrow \infty} \inf _{w \in \mathcal{W}_{\mathcal{A}}} \hat{F}(n !, u, w) \geq \liminf _{n \in \mathbb{N}} \inf _{w \in \mathcal{W}_{\mathcal{A}}} \hat{F}(n, u, w)=F_{\text {hom }}(u) \\
& \quad \geq \inf _{n \in \mathbb{N}} \inf _{w \in \mathcal{W}_{\mathcal{A}}} \hat{F}(n, u, w) \geq \inf _{n \in \mathbb{N}} \inf _{w \in \mathcal{W}_{\mathcal{A}}} \hat{F}(n !, u, w)=\lim _{n \rightarrow \infty} \inf _{w \in \mathcal{W}_{\mathcal{A}}} \hat{F}(n !, u, w) .
\end{aligned}
$$

We conclude that

and, similarly,

$$
F_{\text {hom }}(u)=\inf _{n \in \mathbb{N}} \inf _{w \in \mathcal{W}_{\mathcal{A}}} \hat{F}(n, u, w)=\lim _{n \rightarrow \infty} \inf _{w \in \mathcal{W}_{\mathcal{A}}} \hat{F}(n !, u, w)
$$

$$
f_{\text {hom }}(x, \xi)=\inf _{n \in \mathbb{N}} \inf _{v \in \mathcal{V}_{\mathcal{A}}} \hat{f}(n, x, \xi, v)=\lim _{n \rightarrow \infty} \inf _{v \in \mathcal{V}_{\mathcal{A}}} \hat{f}(n !, x, \xi, v) .
$$

The following lemma is an important ingredient in the proof of Theorem 3.1. 
Lemma 3.4 ( $\mathcal{A}$-free decomposition lemma [18]). Suppose that $\mathcal{A}$ satisfies (1.2), let $D \subset \mathbb{R}^{N}$ be open and bounded, let $1<p<\infty$ and let $\left\{u_{n}\right\} \subset L^{p}\left(D ; \mathbb{R}^{M}\right)$ be a bounded sequence of $\mathcal{A}$-free functions. Then there exists a subsequence $\left\{u_{k(n)}\right\}$ of $\left\{u_{n}\right\}$ and a bounded, $\mathcal{A}$-free, $p$-equiintegrable sequence $\left\{v_{n}\right\} \subset L^{p}\left(D ; \mathbb{R}^{M}\right)$, such that $u_{k(n)}-v_{n} \rightarrow 0$ in $L^{q}$ for every $q \in[1, p)$.

The proof of Theorem 3.1 is divided into establishing the upper and the lower bounds for $\Gamma-\lim F_{\varepsilon}$. We will use the following two technical results. For their proofs, the reader is referred to Appendix B.

Proposition 3.5. Let $f$ satisfy (HO)-(H2), let $\Omega^{\prime} \subset \subset \Omega$ be open, let $\varepsilon_{n} \rightarrow 0^{+}$as $n \rightarrow \infty$, let $\left\{g_{n}\right\} \subset L^{\infty}\left(\Omega, \mathbb{R}^{N}\right)$ be such that $\left\|g_{n}-i d\right\|_{L^{\infty}} \rightarrow 0$, and let $\left\{v_{n}\right\},\left\{w_{n}\right\} \subset$ $L^{p}\left(\Omega ; \mathbb{R}^{M}\right)$ be bounded sequences.

(i) If $\left\{v_{n}\right\},\left\{w_{n}\right\}$ are $p$-equiintegrable and $\left\|v_{n}-w_{n}\right\|_{L^{p}\left(\Omega ; \mathbb{R}^{M}\right)} \rightarrow 0$ then

$$
\lim _{n \rightarrow \infty} \int_{\Omega}\left[f\left(x, \frac{x}{\varepsilon_{n}}, v_{n}(x)\right)-f\left(x, \frac{x}{\varepsilon_{n}}, w_{n}(x)\right)\right] d x=0
$$

and

$$
\lim _{n \rightarrow \infty} \int_{\Omega^{\prime}}\left[f\left(g_{n}(x), \frac{x}{\varepsilon_{n}}, v_{n}(x)\right)-f\left(x, \frac{x}{\varepsilon_{n}}, w_{n}(x)\right)\right] d x=0 .
$$

(ii) If $\left\{w_{n}\right\}$ is p-equiintegrable and $v_{n}-w_{n} \rightarrow 0$ in measure then

$$
\liminf _{n \rightarrow \infty} \int_{\Omega} f\left(x, \frac{x}{\varepsilon_{n}}, v_{n}(x)\right) d x \geq \liminf _{n \rightarrow \infty} \int_{\Omega} f\left(x, \frac{x}{\varepsilon_{n}}, w_{n}(x)\right) d x .
$$

Proposition 3.6. Let $f$ satisfy (HO)-(H2), let $\Omega^{\prime} \subset \subset \Omega$ be open, let $\left\{g_{\nu}\right\} \subset$ $L^{\infty}\left(\Omega^{\prime} \times Q, \mathbb{R}^{N}\right)$, and let $\left\{v_{\nu, n} \mid \nu, n \in \mathbb{N}\right\},\left\{w_{\nu, n} \mid \nu, n \in \mathbb{N}\right\} \subset L^{p}\left(\Omega^{\prime} \times Q ; \mathbb{R}^{M}\right)$ be p-equiintegrable sets.

(i) If $\sup _{n \in \mathbb{N}}\left\|v_{\nu, n}-w_{\nu, n}\right\|_{L^{p}\left(\Omega^{\prime} ; \mathbb{R}^{M}\right)} \rightarrow 0$ and $g_{\nu}(x, y)-x \rightarrow 0$ uniformly in $(x, y) \in$ $\Omega^{\prime} \times Q$ as $\nu \rightarrow \infty$, then

$$
\int_{\Omega^{\prime}} \int_{Q}\left[f\left(g_{\nu}(x), n y, v_{\nu, n}(x, y)\right)-f\left(x, n y, w_{\nu, n}(x, y)\right)\right] d y d x \underset{\nu \rightarrow \infty}{\longrightarrow} 0,
$$

uniformly in $n \in \mathbb{N}$.

(ii) If $\left\|v_{\nu, n}-w_{\nu, n}\right\|_{L^{p}\left(\Omega^{\prime} ; \mathbb{R}^{M}\right)} \rightarrow 0$ as $n \rightarrow \infty$ for every $\nu$ then

$$
\int_{\Omega^{\prime}} \int_{Q}\left[f\left(x, n y, v_{\nu, n}(x, y)\right)-f\left(x, n y, w_{\nu, n}(x, y)\right)\right] d y d x \underset{n \rightarrow \infty}{\longrightarrow} 0,
$$

for every $\nu \in \mathbb{N}$.

Proposition 3.7 (upper bound). Assume that (HO)-(H2) hold. Then for every $n \in$ $\mathbb{N}$, every $\delta>0$, every $u \in \mathcal{U}_{\mathcal{A}}$, and every $w \in \mathcal{W}_{\mathcal{A}}$, there exists a sequence $\left\{u_{\varepsilon}\right\} \subset \mathcal{U}_{\mathcal{A}}$ such that $u_{\varepsilon} \rightarrow u$ in $L^{p}\left(\Omega ; \mathbb{R}^{M}\right)$ as $\varepsilon \rightarrow 0^{+}$, and

$$
\lim _{\varepsilon \rightarrow 0^{+}} \int_{\Omega} f\left(x, \frac{x}{\varepsilon}, u_{\varepsilon}\right) d x \leq \int_{\Omega} \int_{Q} f(x, n y, u(x)+w(x, y)) d y d x+\delta .
$$


Proof. Step 1: Assume first that $u \in C\left(\bar{\Omega} ; \mathbb{R}^{M}\right)$ and $w \in L_{\mathrm{per}}^{p}\left(\mathbb{R}^{N} ; C\left(\bar{\Omega} ; \mathbb{R}^{M}\right)\right) \cap \mathcal{W}_{\mathcal{A}}$ (we write $w(x, y)$ with $y$ being the periodic variable). For fixed $n \in \mathbb{N}$,

$$
g(x, y):=f(x, n y, u(x)+w(x, y)), x \in \bar{\Omega}, y \in \mathbb{R}^{N} \text { (the periodic variable), }
$$

is a function in $L_{\text {per }}^{1}\left(\mathbb{R}^{N} ; C\left(\bar{\Omega} ; \mathbb{R}^{M}\right)\right)$. Moreover, the sequence $\left\{g_{\varepsilon}\right\} \subset L^{1}(\Omega)$, with $g_{\varepsilon}(x):=g\left(x, \frac{x}{n \varepsilon}\right)$, is bounded in $L^{1}$, and by Lemma 2.5, with $v_{\varepsilon}(x):=w\left(x, \frac{x}{\varepsilon}\right)$, we have

$$
\int_{\Omega} f\left(x, \frac{x}{\varepsilon}, u(x)+v_{\varepsilon}(x)\right) d x=\int_{\Omega} g_{\varepsilon}(x) d x \underset{\varepsilon \rightarrow 0^{+}}{\longrightarrow} \int_{\Omega} \int_{Q} g(x, y) d y d x .
$$

In particular, (3.3) holds with equality for $\delta=0$ and $u_{\varepsilon}(x):=u(x)+v_{\varepsilon}(x)$. Note that $\left\{v_{\varepsilon}\right\}$ is a $p$-equiintegrable sequence and, by Proposition 2.4, $v_{\varepsilon} \rightarrow \int_{Q} w(\cdot, y) d y=0$ weakly in $L^{p}$. In addition, still by Proposition 2.4,

$$
\mathcal{A} v_{\varepsilon}=\mathcal{A}_{y_{0}} w\left(\cdot, \frac{\cdot}{n \varepsilon}\right) \underset{\varepsilon \rightarrow 0^{+}}{\rightarrow} \int_{Q} \mathcal{A}_{y_{0}} w\left(\cdot, y_{1}\right) d y_{1}=\mathcal{A} \int_{Q} w\left(\cdot, y_{1}\right) d y_{1}=0
$$

in $L^{p}\left(\Omega ; \mathbb{R}^{L}\right)$, and hence $\mathcal{A} v_{\varepsilon} \rightarrow 0$ in $W^{-1, p}\left(\Omega ; \mathbb{R}^{L}\right)$. Due to (a rescaled version of) Lemma 2.8 , there exists an $\mathcal{A}$-free, $p$-equiintegrable sequence $\left\{\tilde{v}_{\varepsilon}\right\} \subset L^{p}\left(\Omega ; \mathbb{R}^{M}\right)$ such that

$$
\tilde{v}_{\varepsilon}-v_{\varepsilon}=\tilde{v}_{\varepsilon}-w\left(\cdot, \frac{\cdot}{n \varepsilon}\right) \underset{\varepsilon \rightarrow 0^{+}}{\longrightarrow} 0 \text { in } L^{p}\left(\Omega ; \mathbb{R}^{M}\right) .
$$

In particular, $\tilde{v}_{\varepsilon} \rightarrow 0$ in $L^{p}\left(\Omega ; \mathbb{R}^{M}\right)$. By Proposition 3.5 (i) we have

$$
\lim _{\varepsilon \rightarrow 0^{+}} \int_{\Omega} f\left(x, \frac{x}{\varepsilon}, u(x)+\tilde{v}_{\varepsilon}(x)\right) d x=\lim _{\varepsilon \rightarrow 0^{+}} \int_{\Omega} f\left(x, \frac{x}{\varepsilon}, u(x)+v_{\varepsilon}(x)\right) d x
$$

and this, together with (3.4), concludes the proof.

Step 2: The case of a general $u \in \mathcal{U}_{\mathcal{A}}$ and $w \in \mathcal{W}_{\mathcal{A}}$ follows by density and diagonal$\overline{\text { ization }}$ arguments. More precisely, we extend $w$ to $\mathbb{R}^{N}$ by setting $w\left(y_{0}, y_{1}\right):=0$ for $y_{0} \in \mathbb{R}^{N} \backslash \Omega$. Mollifying in $y_{0}$ results in a function $\tilde{w}$ which, in particular, belongs to $L_{\text {per }}^{p}\left(\mathbb{R}^{N} ; C\left(\bar{\Omega} ; \mathbb{R}^{M}\right)\right) \cap \mathcal{W}_{\mathcal{A}}$ and is close to $w$ in the topology of $L^{p}\left(\Omega ; L^{p}\left(\mathbb{R}^{N} ; \mathbb{R}^{M}\right)\right)$. Note that by $(\mathrm{H} 0)$ and (H2), the right hand side of (3.3) is continuous in $w$. Similarly, $u \in \mathcal{U}_{\mathcal{A}}$ can be replaced by a $\tilde{u} \in C\left(\bar{\Omega} ; \mathbb{R}^{M}\right)$ close to $u$ in $L^{p}$ (which in general does not satisfy $\mathcal{A} u=0$ anymore, but this is irrelevant here).

Proposition 3.8 (lower bound 1). Assume that (H0)-(H2) hold. Then for every sequence $\varepsilon_{n} \rightarrow 0^{+}$, every $u \in \mathcal{U}_{\mathcal{A}}$ and every sequence $\left\{u_{n}\right\} \subset \mathcal{U}_{\mathcal{A}}$ with $u_{n} \rightarrow 0$ in $L^{p}\left(\Omega ; \mathbb{R}^{M}\right)$, there exist a family of functions $V=\left\{v_{\nu, n} \mid \nu, n \in \mathbb{N}\right\} \subset \mathcal{U}_{\mathcal{A}}$ such that

$V$ is p-equiintegrable, $v_{\nu, n} \underset{n \rightarrow \infty}{\rightarrow} 0$ weakly in $L^{p}$ for every $\nu \in \mathbb{N}$, and

$$
\liminf _{n \rightarrow \infty} \int_{\Omega} f\left(x, \frac{x}{\varepsilon_{n}}, u+u_{n}\right) d x \geq \sup _{\nu \in \mathbb{N}} \liminf _{n \rightarrow \infty} \int_{\Omega} f\left(x, \nu n x, u+v_{\nu, n}\right) d x .
$$


Proof. Fix $\nu \in \mathbb{N}$. In the following, we repeatedly extract subsequences of $n$ (with all sequences depending on $\nu$ ) without further mentioning and without relabeling $n$. In particular, we may always assume that a limes inferior in $n$ is a limit. For our definition of $v_{\nu, n}$ below, we set $v_{\nu, n}:=0$ if $n$ does not match a value attained by the final ( $\nu$-dependent) subsequence of $n$.

By Lemma 3.4, there exists a $p$-equiintegrable sequence $\left\{\tilde{u}_{n}\right\} \subset \mathcal{U}_{\mathcal{A}}$ such that $\tilde{u}_{n}-$ $u_{n} \rightarrow 0$ in $L^{q}\left(\Omega ; \mathbb{R}^{M}\right)$ for $q<p$ and, in particular, $\tilde{u}_{n} \rightarrow 0$ in $L^{p}$. By Proposition 3.5 (ii), we have that

$$
\lim _{n \rightarrow \infty} \int_{\Omega} f\left(x, \frac{x}{\varepsilon_{n}}, u+u_{n}\right) d x \geq \lim _{n \rightarrow \infty} \int_{\Omega} f\left(x, \frac{x}{\varepsilon_{n}}, u+\tilde{u}_{n}\right) d x .
$$

If $\tilde{k}_{\nu, n}:=\frac{1}{\nu \varepsilon_{n}}$ is a sequence of integers (strictly increasing after selecting a suitable subsequence of $n$ ), then we define $v_{\nu, k}:=u_{n}$ if $k=\tilde{k}_{\nu, n}, v_{\nu, k}:=0$ otherwise. If this is not the case, then for $n \in \mathbb{N}$, let $\left\lfloor\frac{1}{\nu \varepsilon_{n}}\right\rfloor$ denote the largest integer smaller than $\frac{1}{\nu \varepsilon_{n}}$ and let

$$
\theta_{\nu, n}:=\nu \varepsilon_{n}\left\lfloor\frac{1}{\nu \varepsilon_{n}}\right\rfloor \in[0,1] \text {, whence } k_{\nu, n}:=\theta_{\nu, n} \frac{1}{\nu \varepsilon_{n}} \in \mathbb{N}_{0} \text { and } \theta_{\nu, n} \rightarrow 1 \text { as } n \rightarrow \infty \text {. }
$$

Choose an open cube $Y \subset \mathbb{R}^{N}$ which compactly contains $\Omega$. By (a rescaled version of) Lemma 2.8 applied to the sequence $\left\{\tilde{u}_{n}\right\}$, we can find an $\mathcal{A}$-free, bounded, $p$ equiintegrable sequence $\left\{\bar{u}_{n}\right\} \subset L^{p}\left(Y ; \mathbb{R}^{M}\right)$ such that $\bar{u}_{n}-\tilde{u}_{n} \rightarrow 0$ in $L^{p}\left(\Omega ; \mathbb{R}^{M}\right)$ and $\bar{u}_{n} \rightarrow 0$ in $L^{p}\left(Y \backslash \Omega ; \mathbb{R}^{M}\right)$. Using Proposition 3.5 (i) and (3.7) we obtain

$$
\begin{aligned}
\lim _{n \rightarrow \infty} \int_{\Omega} f\left(x, \frac{x}{\varepsilon_{n}}, u+\tilde{u}_{n}\right) d x & =\lim _{n \rightarrow \infty} \int_{\Omega} f\left(x, \frac{x}{\varepsilon_{n}}, u+\bar{u}_{n}\right) d x \\
& =\lim _{n \rightarrow \infty} \int_{\Omega} f\left(x, \nu \frac{k_{\nu, n}}{\theta_{\nu, n}} x, u+\bar{u}_{n}\right) d x .
\end{aligned}
$$

For any fixed $\Omega^{\prime} \subset \subset \Omega, \theta_{\nu, n} \Omega^{\prime} \subset \Omega$ if $n$ is large enough (depending on $\nu$ ). Since $f \geq 0$, a change of variables yields that

$$
\begin{aligned}
& \lim _{n \rightarrow \infty} \int_{\Omega} f\left(x, \nu k_{\nu, n} \frac{1}{\theta_{\nu, n}} x, u(x)+\bar{u}_{n}(x)\right) d x \\
& \geq \liminf _{n \rightarrow \infty} \int_{\theta_{\nu, n} \Omega^{\prime}} f\left(x, \nu k_{\nu, n} \frac{1}{\theta_{\nu, n}} x, u(x)+\bar{u}_{n}(x)\right) d x \\
& =\liminf _{n \rightarrow \infty}\left(\theta_{\nu, n}\right)^{N} \int_{\Omega^{\prime}} f\left(\theta_{\nu, n} x, \nu k_{\nu, n} x, u\left(\theta_{\nu, n} x\right)+\bar{u}_{n}\left(\theta_{\nu, n} x\right)\right) d x \\
& =\liminf _{n \rightarrow \infty} \int_{\Omega^{\prime}} f\left(x, \nu k_{\nu, n} x, u(x)+\bar{u}_{n}\left(\theta_{\nu, n} x\right)\right) d x,
\end{aligned}
$$

where we used Proposition 3.5 (i) and, by (3.6), the facts that $\theta_{\nu, n} \rightarrow 1$ and $u\left(\theta_{\nu, n} \cdot\right) \rightarrow$ $u$ in $L^{p}\left(\Omega^{\prime} ; \mathbb{R}^{M}\right)$. In view of $(3.6)-(3.9)$, we conclude that

$$
\lim _{n \rightarrow \infty} \int_{\Omega} f\left(x, \frac{x}{\varepsilon_{n}}, u_{n}\right) d x \geq \liminf _{n \rightarrow \infty} \int_{\Omega^{\prime}} f\left(x, \nu k_{\nu, n} x, u(x)+\bar{u}_{n}\left(\theta_{\nu, n} x\right)\right) d x .
$$


Letting $\Omega^{\prime}$ approach $\Omega$ such that $\left|\Omega \backslash \Omega^{\prime}\right| \rightarrow 0$, using (H2) and the equiintegrability of $\left\{\left|\bar{u}_{n}\right|^{p}\right\}$, we infer that

$$
\lim _{n \rightarrow \infty} \int_{\Omega} f\left(x, \frac{x}{\varepsilon_{n}}, u_{n}\right) d x \geq \liminf _{n \rightarrow \infty} \int_{\Omega} f\left(x, \nu k_{\nu, n} x, u(x)+v_{\nu, k_{\nu, n}}(x)\right) d x,
$$

with $v_{\nu, k_{\nu, n}}(x):=\bar{u}_{n}\left(\theta_{\nu, n} x\right)$ for $x \in \Omega, \nu \in \mathbb{N}$ and $n \geq n_{0}(\nu)$, where $n_{0}(\nu)$ is chosen large enough such that $\theta_{\nu, n} \geq \frac{1}{2}$ and $\theta_{\nu, n} \Omega \subset Y$ for every $n \geq n_{0}(\nu)$. In particular, $\left\{v_{\nu, k_{\nu, n}} \mid \nu \in \mathbb{N}, n \geq n_{0}(\nu)\right\}$ is $p$-equiintegrable on $\Omega$, just like $\left\{\bar{u}_{n}\right\}$ is p-equiintegrable on $Y$. Moreover, $v_{\nu, k_{\nu, n}} \in \mathcal{U}_{\mathcal{A}}$ and $v_{\nu, k_{\nu, n}} \rightarrow 0$ weakly in $L^{p}$ as $n \rightarrow \infty$.

Proposition 3.9 (lower bound 2). Assume that (HO)-(H2) hold. Then for every $u \in \mathcal{U}_{\mathcal{A}}$, and every family $\left\{v_{\nu, n} \mid \nu, n \in \mathbb{N}\right\} \subset \mathcal{U}_{\mathcal{A}}$ satisfying (3.5),

$$
\begin{aligned}
\liminf _{\nu \rightarrow \infty} \liminf _{n \rightarrow \infty} \int_{\Omega} f\left(x, \nu n x, u+v_{\nu, n}\right) d x \\
\quad \geq \liminf _{n \rightarrow \infty} \inf _{w \in \mathcal{W}_{\mathcal{A}}} \int_{\Omega} \int_{Q} f(x, n y, u(x)+w(x, y)) d y d x .
\end{aligned}
$$

The proof of this Proposition uses a strategy similar to that in Lemma 2.9 in [10], although we work under slightly weaker assumptions on $f$, strongly relying on the p-equiintegrability of $\left\{v_{\nu, n}\right\}$. In particular, we use the so-called unfolding operator $T_{\delta}$ ([11], [10]; see also [12], [26]): For $\delta>0, T_{\delta}: L^{1}\left(\Omega ; \mathbb{R}^{M}\right) \rightarrow L^{1}\left(\Omega ; L_{\text {per }}^{1}\left(\mathbb{R}^{N} ; \mathbb{R}^{M}\right)\right)$ is defined by

$$
T_{\delta}(v)(x, y):=v\left(\delta\left\lfloor\frac{x}{\delta}\right\rfloor+\delta(y-\lfloor y\rfloor)\right) \text { for } x \in \Omega \text { and } y \in \mathbb{R}^{N},
$$

where $v$ is extended by zero outside of $\Omega$ and, as before, for $t \in \mathbb{R},\lfloor t\rfloor$ denotes the largest integer less then or equal to $t$. To arguments in $\mathbb{R}^{N}$ with $N>1,\lfloor\cdot\rfloor$ is applied component-wise. Note that

$$
T_{\delta} v(x, y)=T_{\delta} v\left(\delta\left\lfloor\frac{x}{\delta}\right\rfloor, y\right) \text { for every } \delta>0,(x, y) \in \Omega \times \mathbb{R}^{N} .
$$

Further properties of $T_{\delta}$ are collected in Appendix A.

Proof of Proposition 3.9. Fix $u \in \mathcal{U}_{\mathcal{A}}$ and let $\left\{v_{\nu, n} \mid \nu, n \in \mathbb{N}\right\}$ satisfy (3.5). Moreover, fix $\Omega^{\prime} \subset \subset \Omega$, and for $z \in \mathbb{Z}^{N}$ and $\nu \in \mathbb{N}$ define

$$
Q_{\nu, z}:=\frac{1}{\nu} z+\frac{1}{\nu} Q \subset \mathbb{R}^{N} \text { for } z \in \mathbb{Z}^{N}, Z_{\nu}:=\left\{z \in \mathbb{Z}^{N} \mid Q_{\nu, z} \cap \Omega^{\prime} \neq \emptyset\right\},
$$

and set

$$
I_{\nu, n}:=\int_{\Omega} f\left(x, \nu n x, u+v_{\nu, n}\right) d x .
$$

Note that if $\nu$ is large enough such that the distance of $\Omega^{\prime}$ to $\partial \Omega$ is at least $\frac{\sqrt{N}}{\nu}$, then $Q_{\nu, z} \subset \Omega$ for every $z \in Z_{\nu}$. Since $f \geq 0$, a change of variables and the definition of 
$T_{\frac{1}{\nu}}$ yield

$$
\begin{aligned}
I_{\nu, n} & \geq \sum_{z \in Z_{\nu}} \int_{Q_{\nu, z}} f\left(x, \nu n x, u+v_{\nu, n}\right) d x \\
& =\sum_{z \in Z_{\nu}} \frac{1}{\nu^{N}} \int_{Q} f\left(\frac{z+y}{\nu}, n(z+y), T_{\frac{1}{\nu}}(u)\left(\frac{z}{\nu}, y\right)+T_{\frac{1}{\nu}}\left(v_{\nu, n}\right)\left(\frac{z}{\nu}, y\right)\right) d y \\
& =\sum_{z \in Z_{\nu}} \int_{Q_{\nu, z}} \int_{Q} f\left(\frac{\lfloor\nu x\rfloor+y}{\nu}, n y, T_{\frac{1}{\nu}}(u)\left(\frac{\lfloor\nu x\rfloor}{\nu}, y\right)+T_{\frac{1}{\nu}}\left(v_{\nu, n}\right)\left(\frac{\lfloor\nu x\rfloor}{\nu}, y\right)\right) d y d x
\end{aligned}
$$

where we used (H1) and the facts that $\left|Q_{\nu, z}\right|=\frac{1}{\nu^{N}}$ and $z=\lfloor\nu x\rfloor$ for $x \in Q_{\nu, z}$. Thus, using (3.10) we have that

$$
\begin{aligned}
I_{\nu, n} & \geq \sum_{z \in Z_{\nu}} \int_{Q_{\nu, z}} \int_{Q} f\left(\frac{\lfloor\nu x\rfloor+y}{\nu}, n y, T_{\frac{1}{\nu}}(u)(x, y)+T_{\frac{1}{\nu}}\left(v_{\nu, n}\right)(x, y)\right) d y d x \\
& \geq \int_{\Omega^{\prime}} \int_{Q} f\left(\frac{\lfloor\nu x\rfloor+y}{\nu}, n y, T_{\frac{1}{\nu}}(u)(x, y)+T_{\frac{1}{\nu}}\left(v_{\nu, n}\right)(x, y)\right) d y d x
\end{aligned}
$$

if $\nu$ is sufficiently large such that $\left|\Omega^{\prime} \backslash \bigcup_{z \in Z_{\nu}} Q_{\nu, z}\right|=0$. By Proposition A.2,

$$
U:=\left\{T_{\frac{1}{\nu}}(u)+T_{\frac{1}{\nu}}\left(v_{\nu, n}\right) \mid \nu \in \mathbb{N}, n \in \mathbb{N}\right\} \cup\left\{T_{\frac{1}{\nu}}\left(v_{\nu, n}\right) \mid \nu \in \mathbb{N}, n \in \mathbb{N}\right\},
$$

is a $p$-equiintegrable subset of $L^{p}\left(\Omega^{\prime} \times Q ; \mathbb{R}^{M}\right)$. Moreover, as $\nu \rightarrow \infty, \frac{\lfloor\nu x\rfloor+y}{\nu} \rightarrow$ $x$ uniformly in $(x, y) \in \Omega \times Q$, and $T_{\frac{1}{\nu}}(u) \rightarrow u$ in $L^{p}\left(\Omega^{\prime} \times Q ; \mathbb{R}^{M}\right)$, the latter by Proposition A.1. Hence, (3.11) and Proposition 3.6 (i) imply that

$$
\begin{aligned}
I_{\nu, n} & \geq \sigma_{\nu}+\int_{\Omega^{\prime}} \int_{Q} f\left(x, n y, u(x)+T_{\frac{1}{\nu}}\left(v_{\nu, n}\right)(x, y)\right) d y d x \\
& =\sigma_{\nu}+\sum_{z \in Z_{\nu}} \int_{\Omega^{\prime} \cap Q_{\nu, z}} \int_{Q} f\left(x, n y, u(x)+T_{\frac{1}{\nu}}\left(v_{\nu, n}\right)\left(\frac{z}{\nu}, y\right)\right) d y d x,
\end{aligned}
$$

with an error term $\sigma_{\nu}=\sigma_{\nu}\left(\Omega^{\prime}\right)$ which is independent of $n$ and satisfies $\sigma_{\nu} \rightarrow 0$ as $\nu \rightarrow \infty$ for fixed $\Omega^{\prime}$. Moreover, for fixed $\nu$ and $z \in Z_{\nu}, \hat{v}_{\nu, z, n}(y):=T_{\frac{1}{\nu}}\left(v_{\nu, n}\right)\left(\frac{z}{\nu}, y\right)=$ $v_{\nu, n}\left(\frac{1}{\nu}(z+y)\right), y \in Q$, is a $p$-equiintegrable, $\mathcal{A}$-free sequence in $L^{\nu}\left(Q ; \mathbb{R}^{M}\right)$ with $\hat{v}_{\nu, z, n} \rightarrow 0$ weakly in $L^{p}$ as $n \rightarrow \infty$. By Lemma 2.8 , there exists a $p$-equiintegrable, $\mathcal{A}$-free sequence $\left\{w_{\nu, z, n}\right\} \subset L_{\text {per }}^{p}\left(\mathbb{R}^{N} ; \mathbb{R}^{M}\right)$ such that $\hat{v}_{\nu, z, n}-w_{\nu, z, n} \rightarrow 0$ in $L^{p}\left(Q ; \mathbb{R}^{M}\right)$ as $n \rightarrow \infty$ and $\int_{Q} w_{\nu, z, n}(y) d y=0$. By Proposition 3.6 (ii), we infer that

$$
\begin{aligned}
& \int_{\Omega^{\prime} \cap Q_{\nu, z}} \int_{Q} f\left(x, n y, u(x)+T_{\frac{1}{\nu}}\left(v_{\nu, n}\right)\left(\frac{z}{\nu}, y\right)\right) d y d x \\
& =\tau_{z, n, \nu}+\int_{\Omega^{\prime} \cap Q_{\nu, z}} \int_{Q} f\left(x, n y, u(x)+w_{\nu, z, n}(y)\right) d y d x
\end{aligned}
$$

for every $z \in Z_{\nu}$, where $\lim _{n} \tau_{z, n, \nu}=0$. In view of (3.12), we obtain that

$$
\begin{aligned}
\liminf _{n \rightarrow \infty} I_{\nu, n} & \geq \sigma_{\nu}+\liminf _{n \rightarrow \infty} \sum_{z \in Z_{\nu}} \int_{\Omega^{\prime} \cap Q_{\nu, z}} \int_{Q} f\left(x, n y, u(x)+w_{\nu, z, n}(y)\right) d y d x \\
& =\sigma_{\nu}+\liminf _{n \rightarrow \infty} \int_{\Omega^{\prime}} \int_{Q} f\left(x, n y, u(x)+w_{\nu, n}(x, y)\right) d y d x
\end{aligned}
$$


where

$$
w_{\nu, n}(x, y):=\sum_{z \in Z_{\nu}} \chi_{\Omega^{\prime} \cap Q_{\nu, z}}(x) w_{\nu, z, n}(y),
$$

$\chi_{\Omega^{\prime} \cap Q_{\nu, z}}(x):=1$ if $x \in \Omega^{\prime} \cap Q_{\nu, z}$, and $\chi_{\Omega^{\prime} \cap Q_{\nu, z}}(x):=0$ elsewhere. Clearly, $w_{\nu, n} \in \mathcal{W}_{\mathcal{A}}$, and thus, with

$$
\kappa_{\Omega \backslash \Omega^{\prime}}:=-\sup _{n \in \mathbb{N}} \int_{\Omega \backslash \Omega^{\prime}} \int_{Q} f(x, n y, u(x)) d x d y,
$$

we have that

$$
\begin{aligned}
\liminf _{n \rightarrow \infty} I_{\nu, n} & \geq \kappa_{\Omega \backslash \Omega^{\prime}}+\sigma_{\nu}+\liminf _{n \rightarrow \infty} \int_{\Omega} \int_{Q} f\left(x, n y, u(x)+w_{\nu, n}(x, y)\right) d y d x \\
& \geq \kappa_{\Omega \backslash \Omega^{\prime}}+\sigma_{\nu}+\liminf _{n \rightarrow \infty} \inf _{w \in \mathcal{W}_{\mathcal{A}}} \int_{\Omega} \int_{Q} f(x, n y, u(x)+w(x, y)) d y d x .
\end{aligned}
$$

To conclude let $\nu \rightarrow \infty$ and then let $\Omega^{\prime}$ approach $\Omega$ such that $\left|\Omega \backslash \Omega^{\prime}\right| \rightarrow 0$, using (H2) to ensure that $\kappa_{\Omega \backslash \Omega^{\prime}} \rightarrow 0$ as $\left|\Omega \backslash \Omega^{\prime}\right| \rightarrow 0$.

Proof of Theorem 3.1. Given $u \in \mathcal{U}_{\mathcal{A}}, \varepsilon_{k} \rightarrow 0^{+},\left\{u_{k}\right\} \subset \mathcal{U}_{\mathcal{A}}$ with $u_{k} \rightarrow u$ in $L^{p}\left(\Omega ; \mathbb{R}^{M}\right)$ and setting

$$
J(u):=\liminf _{n \rightarrow \infty} \inf _{w \in \mathcal{W}_{\mathcal{A}}} \int_{\Omega} \int_{Q} f(x, n y, u(x)+w(x, y)) d y d x,
$$

we may assume w.l.o.g. that $\lim \inf \int_{\Omega} f\left(x, \frac{x}{\varepsilon_{k}}, u_{k}\right) d x=\lim \int_{\Omega} f\left(x, \frac{x}{\varepsilon_{k}}, u_{k}\right) d x$, and by Proposition 3.8 and Proposition 3.9, it follows that

$$
\lim _{k \rightarrow \infty} \int_{\Omega} f\left(x, \frac{x}{\varepsilon_{k}}, u_{k}(x)\right) d x \geq J(u) .
$$

Therefore $\Gamma-\liminf F_{\varepsilon}(u) \geq J(u)$.

Conversely, if $\delta>0$ and $\varepsilon_{k} \rightarrow 0^{+}$, and if $n \in \mathbb{N}, w \in \mathcal{W}_{\mathcal{A}}$ are such that

$$
\int_{\Omega} \int_{Q} f(x, n y, u(x)+w(x, y)) d y d x-\delta \leq J(u),
$$

then using Proposition 3.7 we find $\left\{u_{k}\right\} \subset \mathcal{U}_{\mathcal{A}}, u_{k} \rightarrow u$ in $L^{p}\left(\Omega ; \mathbb{R}^{M}\right)$ such that

$$
\lim _{k \rightarrow \infty} \int_{\Omega} f\left(x, \frac{x}{\varepsilon_{k}}, u_{k}(x)\right) d x \leq \int_{\Omega} \int_{Q} f(x, n y, u(x)+w(x, y)) d y d x-\delta \leq J(u)+2 \delta .
$$

Letting $\delta \rightarrow 0$, we conclude that $\Gamma-\lim \sup F_{\varepsilon}(u) \leq J(u)$.

The proof of Corollary 3.2 relies on a measurable selection criterion which is a simplified variant of Theorem III.6 in [9].

Lemma 3.10. Let $Z$ be a separable metric space, let $T$ be a measurable space and let $\Gamma: T \rightarrow 2^{Z}$ be a multifunction such that $\Gamma(t) \subset Z$ is nonempty and open for every $t \in T$, and $\{t \in T \mid z \in \Gamma(t)\}$ is measurable for every $z \in Z$. Then $\Gamma$ admits a measurable selection, i.e., there exists a measurable function $\gamma: T \rightarrow Z$ such that $\gamma(t) \in \Gamma(t)$ for every $t \in T$. 
Proof. Let $Z_{0}:=\left\{z_{k} \mid k \in \mathbb{N}\right\}$ be a countable dense subset of $Z$. Since $\Gamma(t) \neq \emptyset$ and it is open, $\Gamma(t) \cap Z_{0} \neq \emptyset$ for all $t \in T$. We define $\gamma(t):=z_{k(t)}$ if $k(t)$ is the smallest integer such that $z_{k(t)} \in \Gamma(t)$, so that the function $\gamma$ attains values only in the countable set $Z_{0}$. Moreover,

$$
\gamma^{-1}\left(z_{k}\right)=\left\{t \mid z_{k} \in \Gamma(t)\right\} \backslash \bigcup_{j=1}^{k-1}\left\{t \mid z_{j} \in \Gamma(t)\right\}
$$

is measurable for every $k \in \mathbb{N}$, whence $\gamma$ is measurable.

Proof of Corollary 3.2. Fix $u \in \mathcal{U}_{\mathcal{A}}$. Let $\mathcal{V}_{0} \subset \mathcal{V}_{\mathcal{A}}$ be a countable subset which is dense in $\mathcal{V}_{\mathcal{A}}$ with respect to the topology of $L_{\mathrm{per}}^{p}$ (for instance, choose a countable dense subset in $L_{\text {per }}^{p}\left(\mathbb{R}^{N} ; \mathbb{R}^{M}\right)$, and project it onto $\mathcal{V}_{\mathcal{A}}$ using $\left.v \mapsto P(v):=\mathcal{P} v-\int_{Q}(\mathcal{P} v) d y\right)$. For every $n \in \mathbb{N}$, by Lebesgue's Dominated Convergence Theorem and by (H2), we have for a.e. $x \in \Omega$

$$
J_{n}(x, u(x)):=\inf _{v \in \mathcal{V}_{\mathcal{A}}} \int_{Q} f(x, n y, u(x)+v(y)) d y=\inf _{v \in \mathcal{V}_{0}} \int_{Q} f(x, n y, u(x)+v(y)) d y .
$$

In particular, the functions $x \in \Omega \mapsto J_{n}(x, u(x))$ and $x \in \Omega \mapsto f_{\text {hom }}(x, u(x))=$ $\lim _{\inf _{n \rightarrow \infty}} J_{n}(x, u(x))$ are measurable. By Theorem 3.1 and since $w(x, \cdot) \in \mathcal{V}_{\mathcal{A}}$ if $w \in \mathcal{W}_{\mathcal{A}}$, we have

$$
\begin{aligned}
F_{\mathrm{hom}}(u) & =\liminf _{n \rightarrow \infty} \inf _{w \in \mathcal{W}_{\mathcal{A}}} \int_{\Omega} \int_{Q} f(x, n y, u(x)+w(x, y)) d y d x \\
& \geq \liminf _{n \rightarrow \infty} \int_{\Omega}\left(\inf _{v \in \mathcal{V}_{\mathcal{A}}} \int_{Q} f(x, n y, u(x)+v(y)) d y\right) d x \\
& \geq \int_{\Omega}\left(\liminf _{n \rightarrow \infty} \inf _{v \in \mathcal{V}_{\mathcal{A}}} \int_{Q} f(x, n y, u(x)+v(y)) d y\right) d x \\
& =\int_{\Omega} f_{\text {hom }}(x, u(x)) d x
\end{aligned}
$$

where we used Fatou's lemma.

To prove the converse inequality, fix $\delta>0$, and for $m \in \mathbb{N}$ set

$$
\Omega_{m, \delta}:=\bigcup_{n \in\{1, \ldots, m\}}\left\{x \in \Omega \mid J_{n}(x, u(x))<f_{\text {hom }}(x, u(x))+\delta\right\} .
$$

Since $\left|\Omega \backslash \Omega_{m, \delta}\right| \rightarrow 0$ as $m \rightarrow \infty$, there exists $n_{\delta} \in \mathbb{N}$ such that $\left|\Omega \backslash \Omega_{m, \delta}\right| \leq \delta$ for $m \geq n_{\delta}$. Consider the sets

$$
\Gamma_{\delta}(x):=\left\{v \in \mathcal{V}_{\mathcal{A}} \mid \int_{Q} f\left(x, n_{\delta} ! y, u(x)+v(y)\right) d y<f_{\mathrm{hom}}(x, u(x))+\delta\right\}
$$

which are open in $L_{\text {per }}^{p}\left(\mathbb{R}^{N} ; \mathbb{R}^{M}\right)$ (by (H2) and Lebesgue's Dominated Convergence Theorem) and nonempty for $x \in \Omega_{n_{\delta}, \delta}$ (by Remark 3.3, using the fact that $n \mapsto$

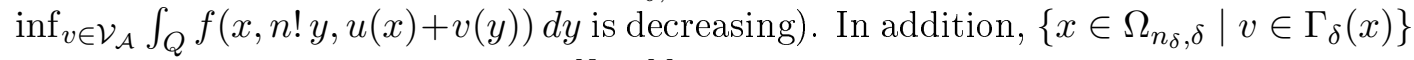
is measurable for every $v \in L_{\text {per }}^{p}\left(\mathbb{R}^{N}, \mathbb{R}^{M}\right)$. We now apply Lemma 3.10 with $T:=\Omega_{n_{\delta}, \delta}$ and $Z:=L_{\mathrm{per}}^{p}\left(\mathbb{R}^{N}, \mathbb{R}^{M}\right)$ to find a measurable selection $\bar{w}: \Omega_{n_{\delta}, \delta} \rightarrow L_{\mathrm{per}}^{p}\left(\mathbb{R}^{N} ; \mathbb{R}^{M}\right)$ of 
$\Gamma_{\delta}$. Moreover, $\bar{w} \in L^{p}\left(\Omega_{\delta}^{\prime} ; L_{\text {per }}^{p}\left(\mathbb{R}^{N} ; \mathbb{R}^{M}\right)\right)$ for a suitable measurable set $\Omega_{\delta}^{\prime} \subset \Omega_{n_{0}, \delta}$ such that

$$
\left|\Omega \backslash \Omega_{\delta}^{\prime}\right| \leq 2 \delta .
$$

For a.e. $x \in \Omega_{\delta}^{\prime}, \bar{w}(x) \in \Gamma_{\delta}(x)$, and thus, since $f \geq 0$, we have

$$
\int_{\Omega_{\delta}^{\prime}} \int_{Q} f\left(x, n_{\delta} ! y, u(x)+\bar{w}(x, y)\right) d y d x \leq \int_{\Omega} f_{\mathrm{hom}}(x, u(x)) d x+\delta|\Omega| .
$$

Extending $\bar{w}(x, \cdot):=0$ for $x \in \Omega \backslash \Omega_{\delta}^{\prime}$, we have that $\bar{w} \in \mathcal{W}_{\mathcal{A}}$ and so,

$$
\begin{aligned}
& \inf _{w \in \mathcal{W}_{\mathcal{A}}} \int_{\Omega} \int_{Q} f\left(x, n_{\delta} ! y, u(x)+w(x, y)\right) d y d x \\
& \leq \int_{\Omega_{\delta}^{\prime}} \int_{Q} f\left(x, n_{\delta} ! y, u(x)+\bar{w}(x, y)\right) d y d x+\int_{\Omega \backslash \Omega_{\delta}^{\prime}} \int_{Q} f\left(x, n_{\delta} ! y, u(x)\right) d y d x \\
& \leq \int_{\Omega} f_{\mathrm{hom}}(x, u(x)) d x+\delta|\Omega|+\int_{\Omega \backslash \Omega_{\delta}^{\prime}} \int_{Q} f\left(x, n_{\delta} ! y, u(x)\right) d y d x .
\end{aligned}
$$

Letting $\delta \rightarrow 0^{+}$, by (H2) and Remark 3.3 we conclude that

$$
F_{\text {hom }}(u) \leq \int_{\Omega} f_{\text {hom }}(x, u(x)) d x
$$

\section{Appendix A Properties of the unfolding operator}

We recall the definition of the unfolding operator (see [11] and [10]; see also [12] and [26]): Let $\Omega \subset \mathbb{R}^{N}$ be open. For $\gamma>0$ and $v \in L^{p}(\Omega)$ ( $v$ extended by zero outside $\Omega$ ), set

$$
T_{\gamma}(v)(x, y):=v\left(\gamma\left\lfloor\frac{x}{\gamma}\right\rfloor+\gamma(y-\lfloor y\rfloor)\right) \text { for } x \in \Omega \text { and } y \in \mathbb{R}^{N},
$$

where as before, $\lfloor\cdot\rfloor$ is defined as the component-wise integer part of its argument. Note that if $v$ has support in $K \subset \subset \mathbb{R}^{N}$ then

$$
\operatorname{supp} T_{\gamma}(v) \subset\left\{x \in \mathbb{R}^{N} \mid \operatorname{dist}(x ; K) \leq \gamma \sqrt{N}\right\} \times \mathbb{R}^{N} .
$$

Indeed, if $\gamma\left\lfloor\frac{x}{\gamma}\right\rfloor+\gamma(y-\lfloor y\rfloor) \in K$ for some $y \in \mathbb{R}^{N}$, then

$\operatorname{dist}(x ; K) \leq\left|x-\gamma\left\lfloor\frac{x}{\gamma}\right\rfloor-\gamma(y-\lfloor y\rfloor)\right|=\gamma\left|\left(\frac{x}{\gamma}-\left\lfloor\frac{x}{\gamma}\right\rfloor\right)-(y-\lfloor y\rfloor)\right| \leq \gamma \operatorname{diam} Q=\gamma \sqrt{N}$.

Proposition A.1. Let $1 \leq p<\infty$. Then for every $\gamma>0, T_{\gamma}: L^{p}(\Omega) \rightarrow L^{p}(\Omega \times Q)$ is linear. Moreover, for every $v \in L^{p}(\Omega)$ (extended by zero outside $\Omega$ ),

$$
\left\|T_{\gamma}(v)\right\|_{L^{p}(\Omega \times Q)} \leq\left\|T_{\gamma}(v)\right\|_{L^{p}\left(\mathbb{R}^{N} \times Q\right)}=\|v\|_{L^{p}\left(\mathbb{R}^{N}\right)}=\|v\|_{L^{p}(\Omega)}
$$

and

$$
\int_{\mathbb{R}^{N}} \int_{Q}\left|v(x)-T_{\gamma}(v)(x, y)\right|^{p} d y d x \rightarrow 0 \quad \text { as } \gamma \rightarrow 0^{+}
$$


Proof. The first equality in (A.2) is a consequence of Fubini's theorem and a change of variables, and the remaining assertions in (A.2) are trivial. For the proof of (A.3), fix $\varepsilon>0$ and choose a sequence $\left\{v_{n}\right\} \subset C_{c}^{\infty}\left(\mathbb{R}^{N}\right)$ with $v_{n} \rightarrow v$ in $L^{p}\left(\mathbb{R}^{N}\right)$. Since $T_{\gamma}$ is linear and by (A.2), there exists $m \in \mathbb{N}$ such that for every $n \geq m$ and every $\gamma>0$,

$$
\left\|v_{n}-v\right\|_{L^{p}\left(\mathbb{R}^{N}\right)}=\left\|T_{\gamma}\left(v_{n}\right)-T_{\gamma}(v)\right\|_{L^{p}\left(\mathbb{R}^{N} \times Q\right)}<\frac{1}{3} \varepsilon .
$$

Hence, with $C_{m}:=\left|\left\{x \in \mathbb{R}^{N} \mid \operatorname{dist}\left(x ; \operatorname{supp} v_{m}\right) \leq \sqrt{N}\right\}\right|^{\frac{1}{p}}$ and using (A.1), for $\gamma \leq 1$ we obtain

$$
\begin{aligned}
\left\|v-T_{\gamma}(v)\right\|_{L^{p}\left(\mathbb{R}^{N} \times Q\right)} & \leq\left\|v_{m}-T_{\gamma}\left(v_{m}\right)\right\|_{L^{p}\left(\mathbb{R}^{N} \times Q\right)}+\frac{2}{3} \varepsilon \\
& \leq C_{m}\left\|v_{m}-T_{\gamma}\left(v_{m}\right)\right\|_{L^{\infty}\left(\mathbb{R}^{N} \times Q\right)}+\frac{2}{3} \varepsilon \\
& =C_{m} \sup _{x \in \mathbb{R}^{N}, y \in Q}\left|v_{m}(x)-v_{m}\left(\gamma\left\lfloor\frac{x}{\gamma}\right\rfloor+\gamma y\right)\right|+\frac{2}{3} \varepsilon .
\end{aligned}
$$

Since $v_{m}$ is uniformly continuous in $\mathbb{R}^{N}$ and

$$
\left.\left|x-\left(\gamma\left\lfloor\frac{x}{\gamma}\right\rfloor+\gamma y\right)\right|=\gamma \mid\left(\frac{x}{\gamma}-\left\lfloor\frac{x}{\gamma}\right\rfloor\right)-y\right) \mid<\gamma \operatorname{diam}(Q)=\gamma \sqrt{N},
$$

by (A.4) we conclude that $\left\|v-T_{\gamma}(v)\right\|_{L^{p}\left(\mathbb{R}^{N} \times Q\right)}<\varepsilon$ for $0<\gamma<\gamma_{0}(m)$ with some $\gamma_{0}(m)>0$ sufficiently small.

Proposition A.2. Let $1 \leq p<\infty$, let $B \subset \mathbb{R}^{N}$ be a bounded set, and let $V \subset L^{p}\left(\mathbb{R}^{N}\right)$ be a p-equiintegrable set of functions with support in $B$. Then $\left\{T_{\gamma} v \mid \gamma \in(0,1], v \in\right.$ $V\} \subset L^{p}\left(\mathbb{R}^{N} \times Q\right)$ is also p-equiintegrable.

Proof. Let $\delta>0$ and choose $\eta>0$ such that

$$
\sup _{v \in V} \int_{F}|v|^{p}<\frac{\delta}{2}
$$

for every measurable $F \subset \mathbb{R}^{N}$ with $|F|<\eta$. Let $t_{0}>>1$ be such that

$$
\sup _{v \in V}\left|\left\{|v(x)|>t_{0}\right\}\right|<\eta
$$

and let $\tau>0$ be such that

$$
\tau t_{0}^{p}<\frac{\delta}{2}
$$

Consider a measurable set $E \subset \mathbb{R}^{N} \times Q$ such that $|E|<\tau$. For $F \subset \mathbb{R}^{N}$ measurable and $\gamma>0$ define

$$
T_{\gamma} F:=\left\{(x, y) \in \mathbb{R}^{N} \times Q \mid \gamma\left\lfloor\frac{x}{\gamma}\right\rfloor+\gamma y \in F\right\} .
$$

Note that

$$
T_{\gamma}\left(\chi_{F}\right)=\chi_{T_{\gamma}(F)}
$$

and for every $v \in V$ and $t \geq 0$,

$$
T_{\gamma}\{|v| \geq t\}=\left\{\left|T_{\gamma} v\right| \geq t\right\} \text { and } T_{\gamma}\{|v|>t\}=\left\{\left|T_{\gamma} v\right|>t\right\} .
$$


Hence, for $v \in V$ we have that

$$
\begin{aligned}
\int_{E}\left|T_{\gamma}(v)\right|^{p} & \leq \int_{E \cap\left\{\left|T_{\gamma}(v)\right| \leq t_{0}\right\}} t_{0}^{p}+\int_{\left\{\left|T_{\gamma}(v)\right|>t_{0}\right\}}\left|T_{\gamma} v\right|^{p} \\
& \leq \tau t_{0}^{p}+\int_{\mathbb{R}^{N} \times Q} \chi_{T_{\gamma}\left\{|v|>t_{0}\right\}}\left|T_{\gamma}(v)\right|^{p} \\
& \leq \frac{\delta}{2}+\int_{\mathbb{R}^{N} \times Q}\left|T_{\gamma}\left(\chi_{\left\{|v|>t_{0}\right\}} v\right)\right|^{p}
\end{aligned}
$$

where we used (A.9), (A.10) and (A.11), in this order, and the fact that $T_{\gamma}(f g)=$ $T_{\gamma}(f) T_{\gamma}(g)$ for functions $f \in L^{\infty}(\Omega), g \in L^{p}\left(\Omega ; \mathbb{R}^{M}\right)$. By (A.2), (A.7) and (A.8), this implies that

$$
\int_{E}\left|T_{\gamma}(v)\right|^{p} \leq \frac{\delta}{2}+\int_{\mathbb{R}^{N}}\left|\chi_{\left\{|v| \geq t_{0}\right\}} v\right|^{p}=\frac{\delta}{2}+\int_{\left\{|v| \geq t_{0}\right\}}|v|^{p}<\delta .
$$

\section{Appendix B Some results on uniform continuity}

Below, we collect several auxiliary results on the continuity of Nemytskii operators associated to the function $f$ introduced in Section 3.

Proposition B.1 (Scorza-Dragoni, e.g. see [20]). Let $\Omega \subset \mathbb{R}^{N}$ be open, let $S_{1} \subset \subset \Omega$, let $S_{2} \subset \subset \mathbb{R}^{M}$ and suppose that $f$ satisfies (HO). Then for every $\delta>0$, there exists a compact set $K_{\delta} \subset Q:=(0,1)^{N}$ such that $\left|Q \backslash K_{\delta}\right|<\delta$ and $f$ is uniformly continuous on $S_{1} \times K_{\delta} \times S_{2}$.

Proposition B.2. Let $1 \leq p<\infty$, assume that (H0)-(H2) hold, let $\lambda \in(0,1]$, let $\Omega^{\prime} \subset \Omega$ be measurable, and let $V \subset L^{p}\left(\Omega^{\prime} ; \mathbb{R}^{M}\right)$ be p-equiintegrable. Then the functions

$$
\boldsymbol{f}_{\lambda}: V \rightarrow L^{1}\left(\Omega^{\prime}\right), \quad \boldsymbol{f}_{\lambda}(v)(x):=f\left(x, \frac{x}{\lambda}, v(x)\right) \text { for } x \in \Omega^{\prime},
$$

are uniformly continuous, uniformly in $\lambda$. Moreover, for every compact $\Omega^{\prime \prime} \subset \Omega$ and $A:=L^{1}\left(\Omega^{\prime} ; \Omega^{\prime \prime}\right) \subset L^{1}\left(\Omega^{\prime} ; \mathbb{R}^{N}\right)$, the functions

$$
\boldsymbol{g}_{\lambda}: A \times V \rightarrow L^{1}\left(\Omega^{\prime}\right), \quad \boldsymbol{g}_{\lambda}(a, v)(x):=f\left(a(x), \frac{x}{\lambda}, v(x)\right) \text { for } x \in \Omega^{\prime},
$$

are uniformly continuous, uniformly in $\lambda$.

Proof. Let $\varepsilon>0$. By the $p$-equiintegrability of $V$ there exists a set $\Omega^{\prime \prime \prime}=\Omega^{\prime \prime \prime}(\varepsilon) \subset \Omega^{\prime}$ such that $\Omega^{\prime \prime \prime} \subset \subset \Omega$ and

$$
\sup _{v \in V} \int_{\Omega^{\prime} \backslash \Omega^{\prime \prime \prime}} f\left(x, \frac{x}{\lambda}, v(x)\right) d x<\frac{\varepsilon}{4} .
$$

Hence, it suffices to show that

$$
\left\|\boldsymbol{f}_{\lambda}\left(v_{1}\right)-\boldsymbol{f}_{\lambda}\left(v_{2}\right)\right\|_{L^{1}\left(\Omega^{\prime \prime \prime}\right)}<\frac{\varepsilon}{2} \text { if }\left\|v_{1}-v_{2}\right\|_{L^{p}\left(\Omega^{\prime \prime \prime} ; \mathbb{R}^{M}\right)}<\delta
$$


for a suitable $\delta=\delta(\varepsilon)>0$ independent of $v_{1}, v_{2} \in V$ and $\lambda \in(0,1]$. This is a special case of the second part of the assertion.

For a proof of the second part of the assertion, let $a_{1}, a_{2} \in A, v_{1}, v_{2} \in V$, and $\lambda \in(0,1]$, and fix $\varepsilon>0$. We want to show that

$$
\left\|\boldsymbol{g}_{\lambda}\left(a_{1}, v_{1}\right)-\boldsymbol{g}_{\lambda}\left(a_{2}, v_{2}\right)\right\|_{L^{1}\left(\Omega^{\prime}\right)}<\varepsilon \text { if }\left\|a_{1}-a_{2}\right\|_{L^{1}\left(\Omega^{\prime} ; \mathbb{R}^{N}\right)}+\left\|v_{1}-v_{2}\right\|_{L^{p}\left(\Omega^{\prime} ; \mathbb{R}^{M}\right)}<\delta,
$$

for a suitable $\delta=\delta(\varepsilon)>0$ independent of $a_{1}, a_{2}, v_{1}, v_{2}$ and $\lambda$. Due to the $p$ equintegrability of $V$ and (H2), there exists $\delta_{1}=\delta_{1}(\varepsilon)>0$ such that for every every $E \subset \Omega^{\prime}$ measurable, and for all $a \in A, v \in V$,

$$
\int_{E}\left|f\left(a(x), \frac{x}{\lambda}, v(x)\right)\right| d x<\frac{\varepsilon}{9}, \text { provided that }|E|<\delta_{1} .
$$

In view of the $p$-equiintegrability of $V$, there exists $R=R(\varepsilon)>0$ large enough such that

$$
\sup _{v \in V}|\{|v| \geq R\}|<\delta_{1} .
$$

Let $L>0$ be sufficiently large so that $\Omega^{\prime} \subset[-L, L]^{N}$. By the periodicity of $f$ with respect to its second variable and by Proposition B.1 applied with $S_{1}:=\Omega^{\prime \prime}$ and $S_{2}:=$ $\bar{B}_{R}(0) \subset \mathbb{R}^{M}$, there exists a compact set $K=K(\varepsilon, L) \subset Q$ and $\delta_{2}=\delta_{2}(\varepsilon, L)>0$, such that

$$
|Q \backslash K|<(2 L+2)^{-N} \delta_{1},
$$

and for every $x_{1}, x_{2} \in \Omega^{\prime \prime}$, every $y \in \mathbb{Z}^{N}+K$ and every $\xi_{1}, \xi_{2} \in \bar{B}_{R}(0)$,

$$
\left|f\left(x_{1}, y, \xi_{1}\right)-f\left(x_{2}, y, \xi_{2}\right)\right|<\frac{\varepsilon}{9\left|\Omega^{\prime}\right|} \text { if }\left|x_{1}-x_{2}\right|+\left|\xi_{1}-\xi_{2}\right|<\delta_{2}
$$

By (B.3), with $m_{\lambda}:=\left\lfloor\frac{L}{\lambda}\right\rfloor+1$, we have that

$$
\begin{aligned}
\left|\Omega^{\prime} \backslash \lambda\left(\mathbb{Z}^{N}+K\right)\right| & \leq\left|[-L, L]^{N} \backslash \lambda\left(\mathbb{Z}^{N}+K\right)\right| \\
& \leq \lambda^{N}\left|\left[-m_{\lambda}, m_{\lambda}\right]^{N} \backslash\left(\mathbb{Z}^{N}+K\right)\right| \\
& =\lambda^{N}\left(2 m_{\lambda}\right)^{N}|Q \backslash K| \\
& \leq(2 L+2)^{N}|Q \backslash K|<\delta_{1} .
\end{aligned}
$$

Finally, there exists $\delta=\delta(\varepsilon)$ such that

$$
\left|\left\{\left|a_{1}-a_{2}\right|+\left|v_{1}-v_{2}\right| \geq \delta_{2}\right\}\right|<\delta_{1} \text { if }\left\|a_{1}-a_{2}\right\|_{L^{1}}+\left\|v_{1}-v_{2}\right\|_{L^{p}}<\delta .
$$

Define

$$
\begin{aligned}
\tilde{S}:=\left[\Omega^{\prime} \cap \lambda\left(\mathbb{Z}^{N}+K\right)\right] & \cap\left\{\left|v_{1}\right|<R\right\} \cap\left\{\left|v_{2}\right|<R\right\} \\
& \cap\left\{\left|a_{1}-a_{2}\right|+\left|v_{1}-v_{2}\right|<\delta_{2}\right\},
\end{aligned}
$$

whence $\Omega^{\prime} \backslash \tilde{S}$ is a union of four sets, each of which has measure less than $\delta_{1}$, due to (B.2), (B.5), and (B.6), respectively. By (B.1) and (B.4), we infer that

$$
\begin{aligned}
\int_{\Omega^{\prime}} \mid & f\left(a_{1}(x), \frac{x}{\lambda}, v_{1}(x)\right)-f\left(a_{2}(x), \frac{x}{\lambda}, v_{2}(x)\right) \mid d x \\
& \leq \frac{8}{9} \varepsilon+\int_{\tilde{S}}\left|f\left(a_{1}(x), \frac{x}{\lambda}, v_{1}(x)\right)-f\left(a_{2}(x), \frac{x}{\lambda}, v_{2}(x)\right)\right| d x<\varepsilon
\end{aligned}
$$

whenever $\left\|a_{1}-a_{2}\right\|_{L^{1}\left(\Omega^{\prime}\right)}+\left\|v_{1}-v_{2}\right\|_{L^{p}\left(\Omega^{\prime}\right)}<\delta$. 
Proposition B.3. Let $1 \leq p<\infty$, assume that (HO)-(H2) hold, let $Q=(0,1)^{N}$, let $\lambda \in(0,1]$, let $\Omega^{\prime} \subset \Omega$ be measurable, and let $\tilde{V} \subset L^{p}\left(\Omega^{\prime} \times Q ; \mathbb{R}^{M}\right)$ be p-equiintegrable. Then for every $\Omega^{\prime \prime} \subset \subset \Omega$ and $\tilde{A}:=L^{1}\left(\Omega^{\prime} \times Q ; \Omega^{\prime \prime}\right) \subset L^{1}\left(\Omega^{\prime} \times Q ; \mathbb{R}^{N}\right)$, the functions

$$
\boldsymbol{h}_{\lambda}: \tilde{A} \times \tilde{V} \rightarrow L^{1}\left(\Omega^{\prime} \times Q\right), \quad \boldsymbol{h}_{\lambda}(a, v)(x, y):=f\left(a(x, y), \frac{y}{\lambda}, v(x, y)\right)
$$

are uniformly continuous, uniformly in $\lambda$.

Proof. This is analogous to the proof of Proposition B.2. We omit the details.

Proof of Proposition 3.5. (i) The first part of (i) follows from the uniform equicontinuity of $\left\{\boldsymbol{g}_{\lambda}\right\}$ obtained in Proposition B.2, with a compact set $\Omega^{\prime \prime}$ satisfying $\Omega^{\prime} \subset \subset \Omega^{\prime \prime} \subset \subset \Omega$. For the second part use the uniform equi-continuity of $\left\{\boldsymbol{f}_{\lambda}\right\}$ obtained in Proposition B.2 with $\Omega^{\prime}:=\Omega$.

(ii) Let $\varepsilon>0$. For $v \in L^{p}(\Omega)$ and $h>0$ consider the truncated function $v^{[h]}:=$ $\max \{\min \{v, h\},-h\}$, whereas for $v \in L^{p}\left(\Omega ; \mathbb{R}^{M}\right), v^{[h]}$ is defined component-wise. Since $\left\{w_{n}\right\}$ is $p$-equiintegrable, so is

$$
W:=\left\{w_{n}: n \in \mathbb{N}\right\} \cup\left\{w_{n}^{[h]}: n \in \mathbb{N}, h>0\right\},
$$

and $w_{n}^{[h]}-w_{n} \rightarrow 0$ in $L^{p}$ as $h \rightarrow \infty$, uniformly in $n \in \mathbb{N}$. Hence, by the first part of (i), there exists $H=H(\varepsilon)>0$ such that

$$
\int_{\Omega}\left|f\left(x, \frac{x}{\varepsilon_{n}}, w_{n}^{[H]}(x)\right)-f\left(x, \frac{x}{\varepsilon_{n}}, w_{n}(x)\right)\right| d x<\frac{\varepsilon}{3},
$$

for every $n \in \mathbb{N}$. Since $\left\{v_{n}^{[H]}\right\}$ and $\left\{w_{n}^{[H]}\right\}$ are $p$-equiintegrable and $w_{n}^{[H]}-v_{n}^{[H]} \rightarrow 0$ in $L^{p}$, again the first part of (i) yields

$$
\int_{\Omega}\left|f\left(x, \frac{x}{\varepsilon_{n}}, v_{n}^{[H]}\right)-f\left(x, \frac{x}{\varepsilon_{n}}, w_{n}^{[H]}(x)\right)\right| d x \underset{n \rightarrow \infty}{\longrightarrow} 0 .
$$

Finally, since $f \geq 0$ and $\left|\left\{v_{n} \neq v_{n}^{[H]}\right\}\right| \rightarrow 0$ as $n \rightarrow \infty$, we have that

$$
\begin{aligned}
\int_{\Omega} f\left(x, \frac{x}{\varepsilon_{n}}, v_{n}\right) d x & -\int_{\Omega} f\left(x, \frac{x}{\varepsilon_{n}}, v_{n}^{[H]}(x)\right) d x \\
& \geq-\int_{\left\{v_{n} \neq v_{n}^{[H]}\right\}} f\left(x, \frac{x}{\varepsilon_{n}}, v_{n}^{[H]}\right) d x \underset{n \rightarrow \infty}{\longrightarrow} 0
\end{aligned}
$$

where we used (H2) and the $p$-equiintegrability of $v_{n}^{[H]}$. Combining (B.7)-(B.9), we infer that

$$
\int_{\Omega} f\left(x, \frac{x}{\varepsilon_{n}}, v_{n}(x)\right) d x \geq \int_{\Omega} f\left(x, \frac{x}{\varepsilon_{n}}, w_{n}(x)\right) d x-\varepsilon
$$

for every $n$ large enough.

Proof of Proposition 3.6. Both assertions are immediate consequences of the uniform equi-continuity of $\left\{\boldsymbol{h}_{\lambda}\right\}$ obtained in Proposition B.3, with a compact set $\Omega^{\prime \prime}$ satisfying $\Omega^{\prime} \subset \subset \Omega^{\prime \prime} \subset \subset \Omega$. 


\section{Acknowledgements}

The authors thank the Center for Nonlinear Analysis (NSF Grants No. DMS-0405343 and DMS-0635983) for its support during the preparation of this paper. The research of I. Fonseca was partially supported by the National Science Foundation under Grants No. DMS-0401763 and DMS-0905778. The research of S. Krömer was supported by the German Research Foundation (DFG) in form of the research fellowship KR 3544/1-1,2.

\section{References}

[1] G. Allaire and M. Briane, Multiscale convergence and reiterated homogenisation, Proc. R. Soc. Edinb., Sect. A 126 (1996), no. 2, 297-342.

[2] Grégoire Allaire, Homogenization and two-scale convergence, SIAM J. Math. Anal. 23 (1992), no. 6, 1482-1518.

[3] Jean-François Babadjian and Margarida Baía, Multiscale nonconvex relaxation and application to thin films, Asymptotic Anal. 48 (2006), no. 3, 173-218.

[4] Margarida Baía and Irene Fonseca, The limit behavior of a family of variational multiscale problems, Indiana Univ. Math. J. 56 (2007), no. 1, 1-50.

[5] L. Berlyand, D. Cioranescu, and D. Golovaty, Homogenization of a GinzburgLandau model for a nematic liquid crystal with inclusions, J. Math. Pures Appl. 84 (2005), no. $1,97-136$.

[6] A. Braides and A. Defranceschi, Homogenization of multiple integrals, Oxford Lecture Series in Mathematics and its Applications, vol. 12, Clarendon Press, Oxford, 1998.

[7] Andrea Braides, Irene Fonseca, and Giovanni Leoni, A-quasiconvexity: Relaxation and homogenization, ESAIM, Control Optim. Calc. Var. 5 (2000), 539-577.

[8] Andrea Braides and Dag Lukkassen, Reiterated homogenization of integral functionals, Math. Models Methods Appl. Sci. 10 (2000), no. 1, 47-71.

[9] C. Castaing and M. Valadier, Convex analysis and measurable multifunctions, Lecture Notes in Mathematics, vol. 580, Springer-Verlag, Berlin-Heidelberg-New York, 1977.

[10] D. Cioranescu, A. Damlamian, and R. De Arcangelis, Homogenization of quasiconvex integrals via the periodic unfolding method, SIAM J. Math. Anal. 37 (2006), no. 5, 1435-1453.

[11] D. Cioranescu, A. Damlamian, and G. Griso, Periodic unfolding and homogenization, C. R., Math., Acad. Sci. Paris 335 (2002), no. 1, 99-104.

[12] , The periodic unfolding method in homogenization, SIAM J. Math. Anal. 40 (2008), no. 4, 585-1620. 
[13] Bernard Dacorogna, Weak continuity and weak lower semi-continuity of nonlinear functionals, Lecture Notes in Mathematics, vol. 922, Springer-Verlag, Berlin-Heidelberg-New York, 1982.

[14] Gianni Dal Maso, An introduction to $\Gamma$-convergence., Progress in Nonlinear Differential Equations and their Applications, no. 8, Birkhäuser, Basel, 1993.

[15] E. De Giorgi and G. Dal Maso, Gamma-convergence and calculus of variations, Mathematical theories of optimization, Proc. Conf., Genova 1981, Lect. Notes Math. 979, 1983, pp. 121-143.

[16] E. De Giorgi and T. Franzoni, Su un tipo di convergenza variazionale., Atti Accad. Naz. Lincei, VIII. Ser., Rend., Cl. Sci. Fis. Mat. Nat. 58 (1975), 842-850 (Italian).

[17] Irene Fonseca and Giovanni Leoni, Modern methods in the calculus of variations. $L^{p}$ spaces., Springer Monographs in Mathematics. New York, NY: Springer, 2007.

[18] Irene Fonseca and Stefan Müller, $\mathcal{A}$-quasiconvexity, lower semicontinuity, and Young measures, SIAM J. Math. Anal. 30 (1999), no. 6, 1355-1390.

[19] Irene Fonseca and Elvira Zappale, Multiscale relaxation of convex functionals., J. Convex Anal. 10 (2003), no. 2, 325-350.

[20] Enrico Giusti, Direct methods in the calculus of variations, World Scientific, Singapore, 2003.

[21] D. Lukkassen, Formulae and bounds connected to optimal design and homogenization of partial differential operators and integrals functionals, Ph.D. thesis, Department of Mathematics, Tromöm University, Norway, 1996.

[22] D. Lukkassen, G. Nguetseng, and P. Wall, Two-scale convergence, Int. J. Pure Appl. Math. 2 (2002), no. 1, 35-86.

[23] François Murat, Compacité par compensation: condition necessaire et suffisante de continuité faible sous une hypothèse de rang constant, Ann. Sc. Norm. Super. Pisa, Cl. Sci., IV. Ser. 8 (1981), 69-102 (French).

[24] Gabriel Nguetseng, A general convergence result for a functional related to the theory of homogenization, SIAM J. Math. Anal. 20 (1989), no. 3, 608-623.

[25] _ Asymptotic analysis for a stiff variational problem arising in mechanics, SIAM J. Math. Anal. 21 (1990), no. 6, 1394-1414.

[26] Augusto Visintin, Some properties of two-scale convergence, Atti Accad. Naz. Lincei, Cl. Sci. Fis. Mat. Nat., IX. Ser., Rend. Lincei, Mat. Appl. 15 (2004), no. $2,93-107$.

[27] _ Towards a two-scale calculus, ESAIM, Control Optim. Calc. Var. 12 (2006), 371-397.

[28] _ Two-scale convergence of first-order operators, Z. Anal. Anwend. 26 (2007), no. 2, 133-164. 